\title{
EXISTENCE AND UNIFORM BOUNDEDNESS OF STRONG SOLUTIONS OF THE TIME-DEPENDENT \\ GINZBURG-LANDAU EQUATIONS OF SUPERCONDUCTIVITY
}

\author{
FOUZI ZAOUCH
}

Received 15 April 2004

The time-dependent Ginzburg-Landau equations of superconductivity with a timedependent magnetic field $\mathbf{H}$ are discussed. We prove existence and uniqueness of weak and strong solutions with $H^{1}$-initial data. The result is obtained under the " $\phi=-\omega(\nabla$. A)" gauge with $\omega>0$. These solutions generate a dynamical process and are uniformly bounded in time.

\section{Introduction}

The time-dependent Ginzburg-Landau equations describing the state of a superconducting material near the critical temperature were derived in 1968 by Gor'kov and Eliashberg [8] by an averaging process of the microscopic BCS theory [2] of superconductivity. They generalize the Ginzburg-Landau model [7] to the nonstationary case. After proper nondimensionalization, the time-dependent Ginzburg-Landau equations consist of a system of nonlinear differential equations for the order-parameter $\psi$, the vector potential $\mathbf{A}$ and the electric potential $\phi$ given by the following:

$$
\begin{aligned}
\eta\left(\frac{\partial}{\partial t}+i \kappa \phi\right) \psi & =-\left(\frac{i}{\kappa} \nabla+\mathbf{A}\right)^{2} \psi+\left(1-|\psi|^{2}\right) \psi \quad \text { in } \Omega \times(0, \infty), \\
\frac{\partial \mathbf{A}}{\partial t}+\nabla \phi & =-\nabla \times \nabla \times \mathbf{A}+\mathbf{J}_{s}+\nabla \times \mathbf{H} \quad \text { in } \Omega \times(0, \infty),
\end{aligned}
$$

where $\mathbf{J}_{s}$ is given by

$$
\mathbf{J}_{s} \equiv \mathbf{J}_{s}(\psi, \mathbf{A})=\frac{1}{2 i \kappa}\left(\psi^{*} \nabla \psi-\psi \nabla \psi^{*}\right)-|\psi|^{2} \mathbf{A}=-\operatorname{Re}\left[\psi^{*}\left(\frac{i}{\kappa} \nabla+\mathbf{A}\right) \psi\right] .
$$

The vector $\mathbf{H}$ represents the (externally) applied magnetic field; it is a given function of position and time. Equations (1.1)-(1.3) are satisfied everywhere in a domain $\Omega$ of $\mathbb{R}^{n}$ ( $n=2$ or 3 ), which is the region occupied by the superconducting material and at all times $t>0$. As usual, $\nabla \equiv \operatorname{grad}, \nabla \cdot \equiv \operatorname{div}, \nabla \times \equiv \operatorname{curl}$ and $\nabla^{2}=\nabla \cdot \nabla \equiv \Delta, i$ is the imaginary unit and a superscript $*$ denotes the complex conjugation. The associated boundary 
conditions are

$$
\mathbf{n} \cdot\left(\frac{i}{\kappa} \nabla+\mathbf{A}\right) \psi+\frac{i}{\kappa} \gamma \psi=0, \quad \mathbf{n} \times(\nabla \times \mathbf{A}-\mathbf{H})=\mathbf{0} \quad \text { on } \partial \Omega,
$$

where $\partial \Omega$ is the boundaryof $\Omega$ and $\mathbf{n}$ the local outer unit normal to $\partial \Omega$. They must be satisfied at all times $t>0$. Henceforth, the term "TDGL Equations" refers to the system of equations (1.1)-(1.4). The unknown functions are $\psi$ a complex-valued function, A taking its values in $\mathbb{R}^{n}$ and $\phi$ a real-valued function. They introduce some physical quantities like the density of the superconducting carriers $|\psi|^{2}$, the supercurrent density $\mathbf{J}_{s}$, the magnetic induction $\mathbf{B}=\nabla \times \mathbf{A}$ and the electric field $\mathbf{E}=\partial_{t} \mathbf{A}-\nabla \phi$. The parameters $\eta$ and $\kappa$ of the model are some physical constants, with $\eta$, a (dimensionless) coefficient friction, and $\kappa$ is the (dimensionless) Ginzburg-Landau parameter. The function $\gamma$ is defined, positive and Lipschitz continuous on $\partial \Omega$; it represents the contact parameter which is zero if the superconducting material is surrounded by vacuum. For more physical meaning of the TDGL equations, see [1] or [21]. On the other hand, the TDGL equations are invariant under the following gauge transformation:

$$
\mathscr{G}_{\chi}:(\psi, \mathbf{A}, \phi) \longrightarrow\left(\tilde{\psi}=\psi \mathrm{e}^{i \kappa \chi}, \tilde{\mathbf{A}}=\mathbf{A}+\nabla \chi, \tilde{\phi}=\phi-\frac{\partial \chi}{\partial t}\right)
$$

here $\chi$ is a given real-valued function (sufficiently smooth) of position and time. In a physical point of view, the physical states of the superconductor describing by $(\psi, \mathbf{A}, \phi)$ and $(\tilde{\psi}, \tilde{\mathbf{A}}, \tilde{\phi})$ are indistinguishable. For more details about gauge invariance, one may consult [5] or [6]. We restrict ourselves to the " $\phi=-\omega(\nabla \cdot \mathbf{A})$ gauge $(\omega>0)$ in which the TDGL equations read

$$
\begin{aligned}
\eta \frac{\partial \psi}{\partial t}=-\left(\frac{i}{\kappa} \nabla+\mathbf{A}\right)^{2} \psi+i \eta \kappa \omega \psi(\nabla \cdot \mathbf{A})+\left(1-|\psi|^{2}\right) \psi & \text { in } \Omega \times(0, \infty), \\
\frac{\partial \mathbf{A}}{\partial t}=-\nabla \times \nabla \times \mathbf{A}+\omega \nabla(\nabla \cdot \mathbf{A})+\mathbf{J}_{s}+\nabla \times \mathbf{H} & \text { in } \Omega \times(0, \infty),
\end{aligned}
$$

where $\mathbf{J}_{s}$ is given by (1.3) and the boundary conditions become

$$
\mathbf{n} \cdot \nabla \psi+\gamma \psi=0, \mathbf{n} \cdot \mathbf{A}=0, \mathbf{n} \times(\nabla \times \mathbf{A}-\mathbf{H})=\mathbf{0} \quad \text { on } \partial \Omega \times(0, \infty) .
$$

For the initial condition, we put

$$
\psi(\cdot, 0)=\psi_{0}, \quad \mathbf{A}(\cdot, 0)=\mathbf{A}_{0} \quad \text { in } \Omega,
$$

where $\psi_{0}$ and $\mathbf{A}_{0}$ are given.

In this paper, we consider the TDGL equations under the gauge choice " $\phi=-\omega(\nabla$. A)" $(\omega>0)$ and in the case of a time dependent magnetic field. In practice, $\mathbf{H}$ is either time independent or time periodic. The question of existence, uniqueness and regularity of weak solutions of the TDGL equations was in particular investigated in $[4,13]$ and [19] for $\mathbf{H}$ time independent and $[6,12,17,22,23]$ for $\mathbf{H}$ time dependent. The long time asymptotic behavior of the solutions of the TDGL equations was mainly settled 
in [6]. The authors in $[6,22]$ assumed among other things that the applied magnetic field satisfies

$$
\int_{0}^{T} \int_{\Omega}|\mathbf{H}(x, t)|^{2} \mathrm{~d} x \mathrm{~d} t<\infty, \quad \int_{0}^{T} \int_{\Omega}\left|\frac{\partial \mathbf{H}}{\partial t}(x, t)\right|^{2} \mathrm{~d} x \mathrm{~d} t<\infty,
$$

for some $T>0$. In this case, the solutions of the TDGL equations generate a dynamical process in some suitable Hilbert spaces, see $[6,22]$. In particular, if $\mathbf{H}$ is time independent, the process becomes a dynamical system enjoying a global attractor. Furthermore, the solutions are attracted to the set of divergence free equilibria, which are the solutions of the time-independent Ginzburg-Landau equations. It is the purpose here to deal with the situation in which $\mathbf{H}$ satisfies not completely (1.10). More precisely, we assume that $\partial \mathbf{H} / \partial t$ may be not integrable in a neighborhood of the origin instant $t=0$. One of the main objective of this article is to generalize many of the existence results concerning the TDGL equations by showing existence of global (in time) strong solutions. Such kind of solutions have not been previously studied in the literature. Furthermore, we perform our analysis by developing and improving a number of estimates on the solutions involving Sobolev imbeddings. In particular, we stress the fact that the solutions are Hölder continuous and uniform bounded in time. Finally, we mention that the a-priori bounds on the solutions obtained here improve those established in [22]. The paper is organized as follows. In Section 2, we introduce preliminary materials and recall some basic results for use in subsequent sections. Section 3 contains results on the existence and uniqueness of weak and strong solutions for the TDGL equations. Some aspects on the dynamics of the solutions are discussed in Section 4. In the last section, we establish global a-priori estimates on the solutions thus obtaining existence and uniform boundedness for all time $t \geq 0$.

\section{Functional formulation}

We assume that $\Omega$ is a bounded domain in $\mathbb{R}^{n}(n=2$ or 3$)$ with a boundary $\partial \Omega$ of class $C^{1,1}$. In the sequel, we take $n=3$ since the case $n=2$ is similar. The function $\gamma$ is defined and Lipschitz continuous on $\partial \Omega$ and $\gamma(x) \geq 0$ for $x \in \partial \Omega$. Throughout, for $p \geq 1$, $L^{p}(\Omega)$ will denote the usual Lebesgue space, with the norm $\|\cdot\|_{p},\langle\cdot, \cdot\rangle$ is the usual innerproduct in $L^{2}(\Omega)$. For nonnegative integer $m$, we will denote by $H^{m}(\Omega)$ the usual Sobolev space, with norm $\|\cdot\|_{H^{m}}$. In the case of nonintegers $m, H^{m}(\Omega)$ is the fractional Sobolev space defined by interpolation. The corresponding spaces of complex-valued functions will be denoted by $\mathscr{L}^{P}(\Omega)$ and $\mathscr{H}^{m}(\Omega)$ and the corresponding spaces of vector valued functions will be denoted by $\mathbf{L}^{p}(\Omega)$ and $\mathbf{H}^{m}(\Omega)$. Without any possible ambiguity, we use the same symbol $\|\cdot\|_{p}$ to indicate the norms in $\mathscr{L}^{p}(\Omega)$ and $\mathbf{L}^{p}(\Omega)$, and the inner-product for $p=2$ is defined in the usual way. We sometimes use $\|\cdot\|_{X}$ to denote the norm defined on a Banach space $X$. At each time $t \geq 0$, we assume that $\mathbf{H} \in \mathbf{L}^{2}(\Omega)$ and consider the vector $\mathbf{A}_{\mathbf{H}} \in \mathbf{H}^{1}(\Omega)$ defined to be the weak solution of the strongly elliptic problem

$$
\begin{array}{lll}
\nabla \cdot \mathbf{A}_{\mathbf{H}}=0, & \nabla \times \nabla \times \mathbf{A}_{\mathbf{H}}=\nabla \times \mathbf{H} & \text { in } \Omega, \\
\mathbf{n} \cdot \mathbf{A}_{\mathbf{H}}=0, & \mathbf{n} \times\left(\nabla \times \mathbf{A}_{\mathbf{H}}-\mathbf{H}\right)=0 & \text { on } \partial \Omega .
\end{array}
$$


At each fixed time $t \geq 0$, the mapping $\mathbf{H} \in \mathbf{L}^{2}(\Omega) \longmapsto \mathbf{A}_{\mathbf{H}} \in \mathbf{H}^{1}(\Omega)$ is linear and continuous, see [6]. Moreover, the vector $\mathbf{A}_{\mathbf{H}}$ turns the boundary condition in the right hand side of (1.8) into a homogenous one. In fact, by setting

$$
\mathbf{A}^{\prime}:=\mathbf{A}-\mathbf{A}_{\mathbf{H}}
$$

and substituting in (1.6)-(1.9) one obtain the following system:

$$
\begin{aligned}
\eta \frac{\partial \psi}{\partial t}=-\left(\frac{i}{\kappa} \nabla+\mathbf{A}^{\prime}+\mathbf{A}_{\mathbf{H}}\right)^{2} \psi+i \eta \kappa \omega \psi\left(\nabla \cdot \mathbf{A}^{\prime}\right)+\left(1-|\psi|^{2}\right) \psi & \text { in } \Omega \times(0, \infty), \\
\frac{\partial \mathbf{A}^{\prime}}{\partial t}=-\nabla \times \nabla \times \mathbf{A}^{\prime}+\omega \nabla\left(\nabla \cdot \mathbf{A}^{\prime}\right)+\mathbf{J}_{s}^{\prime}-|\psi|^{2} \mathbf{A}_{\mathbf{H}}-\frac{\partial \mathbf{A}_{\mathbf{H}}}{\partial t} & \text { in } \Omega \times(0, \infty), \\
\mathbf{n} \cdot \nabla \psi+\gamma \psi=0, \mathbf{n} \cdot \mathbf{A}^{\prime}=0, \quad \mathbf{n} \times\left(\nabla \times \mathbf{A}^{\prime}\right)=\mathbf{0} & \text { on } \partial \Omega \times(0, \infty) .
\end{aligned}
$$

The supplemented initial condition is

$$
\psi(\cdot, 0)=\psi_{0}, \quad \mathbf{A}^{\prime}(\cdot, 0)=\mathbf{A}_{0}^{\prime}=\mathbf{A}_{0}-\mathbf{A}_{\mathbf{H}}(0) \text { in } \Omega .
$$

Here $\mathbf{J}_{s}^{\prime}=\mathbf{J}_{s}\left(\psi, \mathbf{A}^{\prime}\right)$ is given by the expression in (1.3). On the other hand, to fix the time dependence of the functions entering equations (2.4)-(2.7), we introduce the following spaces. For any given $T>0, p \geq 1$ and any given Banach space $X$, we put

$$
\begin{aligned}
& L^{p}(0, T ; X) \\
& =\left\{u: t \in(0, T) \rightarrow u(\cdot, t) \in X \text { measurable, and } \int_{0}^{T}\|u(\cdot, t)\|_{X}^{p} \mathrm{~d} t<\infty\right\}, \\
& L^{\infty}(0, T ; X) \\
& =\left\{u: t \in(0, T) \rightarrow u(\cdot, t) \in X \text { measurable, and ess } \sup _{0<t<T}\|u(\cdot, t)\|_{X}<\infty\right\}, \\
& W^{1, p}(0, T ; X) \\
& =\left\{u \in L^{p}(0, T ; X) \text { absolutely continuous such that } \frac{\partial u}{\partial t} \in L^{p}(0, T ; X)\right\} .
\end{aligned}
$$

The spaces $W^{m, p}(0, T ; X)$ are defined in similar ways. $C([0, T] ; X)$ denotes the space of continuously $\mathrm{X}$-valued functions defined in $[0, T]$ and $C^{\theta}([0, T], X)$ the family of all Hölder continuous $\mathrm{X}$-valued functions with exponent $\theta,(0<\theta<1)$. One may consult [3] for details about these notions.

In order to reformulate the system (2.4)-(2.7) into an equivalent abstract initial value problem, we consider the solutions $\psi$ and $\mathbf{A}^{\prime}$ as a vector representing the pair $u=\left(\psi, \mathbf{A}^{\prime}\right)$ and adopt the following notation

$$
\mathbb{L}^{p}(\Omega)=\mathscr{L}^{p}(\Omega) \times \mathbf{L}^{p}(\Omega), \quad \mathbb{H}^{s}(\Omega)=\mathscr{H}^{s}(\Omega) \times \mathbf{H}^{s}(\Omega) .
$$

If no ambiguity is possible, we use the same symbol $\|\cdot\|_{p}$ for the norm of $\llbracket^{p}(\Omega)$. Let $\mathscr{A}$ be the linear selfadjoint operator in $\mathbb{L}^{2}(\Omega)$ associated with the following quadratic form:

$$
\mathscr{2}_{\omega}(u)=\int_{\Omega}\left[\frac{1}{\eta \kappa^{2}}|\nabla \psi|^{2}+\omega(\nabla \cdot \mathbf{A})^{2}+|\nabla \times \mathbf{A}|^{2}\right] d x+\frac{1}{\eta \kappa^{2}} \int_{\partial \Omega} \gamma|\psi|^{2} d \sigma(x),
$$


on the domain

$$
\mathscr{D}\left(\mathscr{Q}_{\omega}\right)=\left\{u=(\psi, \mathbf{A}) \in \mathbb{U}^{1}(\Omega): \mathbf{n} \cdot \mathbf{A}=0 \quad \text { on } \partial \Omega\right\} .
$$

Some general results on second-order elliptic differential operators show that the operator $\mathscr{A}$ is sectorial. We omit the details; one may consult [10]. Hence $-\mathscr{A}$ generates a holomorphic semigroup $\left(\mathrm{e}^{-\mathscr{A} t}\right)_{t \geq 0}$ in $\mathbb{L}^{2}(\Omega)$ and the fractional powers $\mathscr{A}^{\alpha}$ of $\mathscr{A}$ are well defined. Interpolation theory shows that the domain $\mathscr{D}\left(\mathscr{A}^{\alpha}\right)$ is a closed linear subspace of $\mathbb{H}^{2 \alpha}(\Omega)$ for $0<\alpha<1$ and in particular $\mathscr{D}\left(\mathscr{A}^{1 / 2}\right)=\mathscr{D}\left(\mathscr{2}_{\omega}\right)$. On the other hand, it is possible to extend the operator $\mathscr{A}$ to the Banach space $\mathbb{L}^{p}(\Omega)$, with $1<p<\infty$. We will use the symbol $\mathscr{A}_{p}$ for a more general definition of $\mathscr{A}$ as an unbounded linear operator in $\mathbb{L}^{p}(\Omega)$. In this case, $\mathscr{A}_{p}$ is also sectorial and so $-\mathscr{A}_{p}$ generates a holomorphic semigroup in $\mathbb{L}^{p}(\Omega)$ (see [10] and [14] for more details). The remaining nonlinear term in the system of equations (2.4)-(2.5) is defined as follows:

$$
\begin{aligned}
& \Gamma(t, \psi, \mathbf{A})=\frac{1}{\eta}[-\frac{2 i}{\kappa}(\nabla \psi) \cdot\left(\mathbf{A}+\mathbf{A}_{\mathbf{H}}\right)-\frac{i}{\kappa}\left(1-\eta \kappa^{2} \omega\right) \psi(\nabla \cdot \mathbf{A}) \\
&\left.-\psi\left|\mathbf{A}+\mathbf{A}_{\mathbf{H}}\right|^{2}+\left(1-|\psi|^{2}\right) \psi\right] \\
& \mathbf{F}(t, \psi, \mathbf{A})=\mathbf{J}_{s}(\psi, \mathbf{A})-|\psi|^{2} \mathbf{A}_{\mathbf{H}}-\frac{\partial \mathbf{A}_{\mathbf{H}}}{\partial t}
\end{aligned}
$$

for all $(\psi, \mathbf{A}) \in \mathbb{U}^{1}(\Omega)$ and $t>0$. Observe that the mapping $\mathscr{F}:=(\Gamma, \mathbf{F})$ maps $[0, T] \times$ $\mathbb{M}^{1}(\Omega)$ in $\mathbb{L}^{3 / 2}(\Omega)$. Therefore, the semigroup $\left\{\mathrm{e}^{-\mathscr{A} t}: t \geq 0\right\}$ does not act on $\mathscr{F}$. For this reason, we will take instead of $\mathscr{A}$ the operator $\mathscr{B}=\mathscr{A}_{3 / 2}$. As mentioned above, $\mathscr{B}$ is the extension of the operator $\mathscr{A}$ to the Banach space $\mathbb{L}^{3 / 2}(\Omega)$. Let $u_{0} \in \mathbb{M}^{1}(\Omega)$, then equations (2.4)-(2.7) are equivalent to an evolutionary system, namely,

$$
\left\{\begin{array}{l}
\frac{\mathrm{d} u}{\mathrm{~d} t}+\mathscr{B} u=\mathscr{F}(t, u(t)) \quad \text { for } t>0, \\
u(0)=u_{0} .
\end{array}\right.
$$

$u_{0}$ being in $\mathbb{Q}^{1}(\Omega)$, we are interested in the so called mild solution of problem (2.16), namely a continuous function $u:[0, T] \rightarrow \mathbb{M}^{1}(\Omega)(T>0$ is given $)$ such that

$$
u(t)=\mathrm{e}^{-\mathscr{A} t} u_{0}+\int_{0}^{t} \mathrm{e}^{-\mathscr{B}(t-s)} \mathscr{F}(s, u(s)) \mathrm{d} s \quad \text { for } 0 \leq t \leq T .
$$

\section{Existence and uniqueness}

In this section, we shall assume that the magnetic field $\mathbf{H}$ satisfies the following assumptions:

$$
\begin{gathered}
\mathbf{H} \in L^{\infty}\left(0, T ; \mathbf{L}^{2}(\Omega)\right) \cap W^{1,2}\left(\tau, T ; \mathbf{L}^{2}(\Omega)\right) \quad \forall 0<\tau \leq T \\
\lim _{t \rightarrow 0^{+}}\left(t^{\mu}\left\|\frac{\partial \mathbf{H}}{\partial t}(t)\right\|_{2}\right)=0, \quad \text { for some } \mu<1 .
\end{gathered}
$$


Observe that if $\mu<1 / 2$, conditions (3.1) and (3.2) imply (1.10). This case is investigated in [6] and [22]. We then restrict ourselves in the sequel to the case $1 / 2 \leq \mu<1$. Moreover, (3.1) and (3.2) imply $\mathbf{H} \in C\left([0, T], \mathbb{L}^{2}(\Omega)\right)$. Before we investigate the question of existence and uniqueness of mild solutions of the associated initial value problem (2.16), we need to collect some a-priori informations on the solutions. Let us define the following mapping:

$$
\mathscr{F}^{\prime}(t, u)=\mathscr{F}(t, u)+\left(0, \frac{\partial \mathbf{A}_{\mathbf{H}}}{\partial t}(t)\right) \quad \text { for a.e } 0<t<T, \quad u=(\psi, \mathbf{A}) \in \mathbb{H}^{1}(\Omega) \text {. }
$$

The first lemma concerns Hölder continuity of the mild solutions.

LEMMA 3.1. Let $T>0$ and $v \in L^{\infty}\left(0, T ; \mathbb{T}^{1}(\Omega)\right)$ and set

$$
\mathscr{I}_{\mathbf{H}}(t)=\int_{0}^{t} \mathrm{e}^{-\mathscr{B}(t-s)} \mathscr{F}^{\prime}(s, v(s)) \mathrm{d} s \quad \text { for } t \in[0, T]
$$

Then for every $0<\theta<1 / 4$

$$
\Phi_{\mathbf{H}} \in C^{\theta}\left([0, T], \mathbb{H}^{1}(\Omega)\right) .
$$

Proof. Since $\Omega$ is smooth enough and the domain of $\mathscr{B}$ is embedded in $W^{2,3 / 2}(\Omega)$, we have for all $3 / 4<\gamma<1$ the fractional spaces $\mathscr{D}\left(\mathscr{B}^{\gamma}\right)$ of the operator $\mathscr{B}$ are continuously embedded in $\mathbb{W}^{1}(\Omega)$, that is,

$$
\|v\|_{\mathbb{1}^{1}} \leq c_{\gamma}\left\|\mathscr{B}^{\gamma} v\right\|_{3 / 2} \quad \forall v \in \mathscr{D}\left(\mathscr{B}^{\gamma}\right),
$$

for some positive constant $c_{\gamma}$ depending on $\gamma \in(3 / 4,1)$. Moreover, we have

$$
\begin{gathered}
\left\|\mathscr{B}^{\alpha} \mathrm{e}^{-\mathscr{B} t} v\right\|_{3 / 2} \leq C_{\alpha} t^{-\alpha} \mathrm{e}^{-\delta t}\|v\|_{3 / 2} \quad \forall t>0, \forall \alpha \geq 0, \forall v \in \mathbb{E}^{3 / 2}(\Omega), \\
\left\|\left(\mathrm{e}^{-\mathscr{B} t}-I\right) v\right\|_{3 / 2} \leq K_{\alpha} t^{\alpha}\left\|\mathscr{B}^{\alpha} v\right\|_{3 / 2} \quad \forall t \geq 0, \forall 0<\alpha \leq 1, \forall v \in \mathscr{D}\left(\mathscr{B}^{\alpha}\right),
\end{gathered}
$$

where $C_{\alpha}$ and $K_{\alpha}$ are some positive constants independent from $t$. The constant $\delta$ depends only on the semigroup. For more details, we refer to $[10,14]$.

$\mathbf{H}$ being in $L^{\infty}\left(0, T ; \mathbf{L}^{2}(\Omega)\right)$ implies that $\mathbf{A}_{\mathbf{H}} \in L^{\infty}\left(0, T ; \mathbf{H}^{1}(\Omega)\right)$. On the other hand, we have $v \in L^{\infty}\left(0, T ; \mathbb{\boxplus}^{1}(\Omega)\right)$. Therefore, substituting in (2.14) and (2.15) and using standard arguments imply

$$
\left\|\mathscr{F}^{\prime}(t, v(t))\right\|_{3 / 2}<\infty \quad \text { for a.e } 0 \leq t \leq T \text {. }
$$

Let $h \geq 0$ and $t \in[0, T)$ be such that $t+h \in[0, T]$. We write

$$
\begin{gathered}
\mathscr{I}_{\mathbf{H}}(t+h)-\mathscr{I}_{\mathbf{H}}(t)=I_{1}+I_{2}, \\
I_{1}=\int_{0}^{t}\left(\mathrm{e}^{-\mathscr{B} h}-I\right) \mathrm{e}^{-\mathscr{B}(t-s)} \mathscr{F}^{\prime}(s, v(s)) \mathrm{d} s, \\
I_{2}=\int_{t}^{t+h} \mathrm{e}^{-\mathscr{B}(t+h-s)} \mathscr{F}^{\prime}(s, v(s)) \mathrm{d} s .
\end{gathered}
$$


Let $0<\theta<1 / 4$ and $3 / 4<\gamma<1-\theta$. We estimate each of the two terms separately. For the first one, using (3.6), (3.7), and (3.8) yields

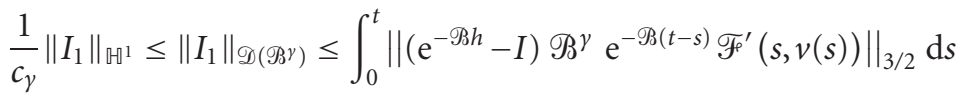

$$
\begin{aligned}
& \leq K_{\theta} h^{\theta} \int_{0}^{t}\left\|\mathscr{B}{ }^{\theta+\gamma} \mathrm{e}^{-\mathscr{B}(t-s)} \mathscr{F}^{\prime}(s, v(s))\right\|_{3 / 2} \mathrm{~d} s \\
& \leq K_{\theta} C_{\theta+\gamma} h^{\theta} \int_{0}^{t}(t-s)^{-(\theta+\gamma)} \mathrm{e}^{-\delta(t-s)}\left\|\mathscr{F}^{\prime}(s, v(s))\right\|_{3 / 2} \mathrm{~d} s \\
& \leq K_{\theta} C_{\theta+\gamma} h^{\theta}\left(\text { ess } \sup _{0 \leq t \leq T}\left\|\mathscr{F}^{\prime}(s, v(s))\right\|_{3 / 2}\right) \int_{0}^{T} s^{-(\theta+\gamma)} \mathrm{e}^{-\delta s} \mathrm{~d} s .
\end{aligned}
$$

Now to estimate $I_{2}$ we have from (3.6), (3.7) and Hölder's inequality

$$
\begin{aligned}
& \frac{1}{c_{\gamma}}\left\|I_{1}\right\|_{\mathbb{Q}^{1}} \leq\left\|I_{2}\right\|_{\mathscr{D}\left(\mathscr{B}^{\gamma}\right)} \leq \int_{0}^{h}\left\|\mathscr{B}^{\gamma} \mathrm{e}^{-\mathscr{B} s} \mathscr{F}^{\prime}(t+h-s, v(t+h-s))\right\|_{3 / 2} \mathrm{~d} s \\
& \leq C_{\gamma} \int_{0}^{h} s^{-\gamma} \mathrm{e}^{-\delta s}\left\|\mathscr{F}^{\prime}(t+h-s, v(t+h-s))\right\|_{3 / 2} \mathrm{~d} s \\
& \leq C_{\gamma}\left(\text { ess } \sup _{0 \leq s \leq T}\left\|\mathscr{F}^{\prime}(s, v(s))\right\|_{3 / 2}\right) \int_{0}^{h} s^{-\gamma} \mathrm{e}^{-\delta s} \mathrm{~d} s \\
& \leq C_{\gamma} h^{\theta}\left(\operatorname{ess} \sup _{0 \leq s \leq T}\left\|\mathscr{F}^{\prime}(s, v(s))\right\|_{3 / 2}\right)\left(\int_{0}^{T} s^{-\gamma / 1-\theta} \mathrm{e}^{-(\delta / 1-\theta) s} \mathrm{~d} s\right)^{1-\theta} .
\end{aligned}
$$

The remaining case is similar. Indeed, let $h \geq 0$ and $t \in(0, T]$. It suffices to write $t^{\prime}=t-h$ to obtain

$$
\mathscr{I}_{\mathbf{H}}(t-h)-\mathscr{I}_{\mathbf{H}}(t)=-\left(\mathscr{I}_{\mathbf{H}}\left(t^{\prime}+h\right)-\mathscr{I}_{\mathbf{H}}\left(t^{\prime}\right)\right),
$$

and so we have to argue exactly as above to complete the proof of the lemma.

Consider now the initial value problem

$$
\left\{\begin{array}{l}
\frac{\mathrm{d} v}{\mathrm{~d} t}+\mathscr{B} v=\mathscr{F}^{\prime}(t, v(t)) \quad \text { for } t>0 \\
v(0)=v_{0}
\end{array}\right.
$$

which coincides with the problem (2.16) in the case of $\mathbf{H}$ time independent. We need for later purpose to look for existence and uniqueness of mild solutions of problem (3.14), that is,

$$
v(t)=\mathrm{e}^{-\mathscr{A} t} v_{0}+\int_{0}^{t} \mathrm{e}^{-\mathscr{B}(t-s)} \mathscr{F}^{\prime}(s, v(s)) \mathrm{d} s \quad \text { for } 0 \leq t \leq T .
$$

More precisely, we investigate the question of existence and uniqueness of strong solutions of the initial value problem problem (3.14). In general cases of parabolic equations, the problem of finding strong solutions requires some assumptions on local Hölder and Lipschitz continuity of the nonlinearity. In fact, we have the following. 
Theorem 3.2. Assume that the magnetic field $\mathbf{H}$ satisfies

$$
\mathbf{H} \in L^{\infty}\left(0, T ; \mathbf{L}^{2}(\Omega)\right) \cap W^{1,2}\left(\tau, T ; \mathbf{L}^{2}(\Omega)\right) \quad \forall \tau>0 .
$$

Then for each initial data $v_{0} \in \mathscr{D}\left(\mathscr{A}^{1 / 2}\right)$ the problem (3.14) possesses a unique strong solution $v=\left(\psi, \mathbf{A}^{\prime}\right)$ such that

$$
v \in C\left([0, T], \mathbb{M}^{1}(\Omega)\right) \cap C^{\theta}\left([\tau, T], \mathbb{H}^{1}(\Omega)\right) \quad \forall 0<\tau \leq T, 0<\theta<1 / 4 .
$$

Proof. The proof of the Theorem 3.2 uses the Hölder's inequalities

$$
\begin{aligned}
\|f g\|_{3 / 2} & \leq\|f\|_{2}\|g\|_{6} \quad \forall f \in L^{2}(\Omega), \forall g \in L^{6}(\Omega), \\
\|f g h\|_{3 / 2} & \leq\|f\|_{3}\|g\|_{6}\|h\|_{6} \quad \forall f \in L^{3}(\Omega), \forall g, h \in L^{6}(\Omega) .
\end{aligned}
$$

Let $B_{R}$ be any ball in the space $\mathbb{H}^{1}(\Omega)$ of radius $R$ and centered at the origin. For any $v_{1}=\left(\varphi_{1}, \mathbf{B}_{1}\right), v_{2}=\left(\varphi_{2}, \mathbf{B}_{2}\right)$ in $B_{R}$ and $t_{1}, t_{2} \in[0, T]$, we have

$$
\left\|\mathscr{F}^{\prime}\left(t_{1}, v_{1}\right)-\mathscr{F}^{\prime}\left(t_{2}, v_{2}\right)\right\|_{3 / 2} \leq\left\|\mathscr{F}^{\prime}\left(t_{1}, v_{1}\right)-\mathscr{F}^{\prime}\left(t_{1}, v_{2}\right)\right\|_{3 / 2}+\left\|\mathscr{F}^{\prime}\left(t_{1}, v_{2}\right)-\mathscr{F}^{\prime}\left(t_{2}, v_{2}\right)\right\|_{3 / 2}
$$

First we have for all $t \in[0, T]$

$$
\begin{aligned}
\left\|\mathscr{F}^{\prime}\left(t, v_{1}\right)-\mathscr{F}^{\prime}\left(t, v_{2}\right)\right\|_{3 / 2} \leq & \left\|\Gamma\left(t, \varphi_{1}, \mathbf{B}_{1}\right)-\Gamma\left(t, \varphi_{2}, \mathbf{B}_{2}\right)\right\|_{3 / 2} \\
& +\left\|\mathbf{J}_{s}\left(\varphi_{1}, \mathbf{B}_{1}\right)-\mathbf{J}_{s}\left(\varphi_{2}, \mathbf{B}_{2}\right)\right\|_{3 / 2} \\
& +\left\|\left(\left|\varphi_{1}\right|^{2}-\left|\varphi_{2}\right|^{2}\right) \mathbf{A}_{\mathbf{H}}(t)\right\|_{3 / 2} .
\end{aligned}
$$

Each term in the expression of $\Gamma$ and $\mathbf{J}_{s}$ will be estimated separately. First we have

$$
\begin{aligned}
\left\|\nabla \varphi_{1} \cdot \mathbf{B}_{1}-\nabla \varphi_{2} \cdot \mathbf{B}_{2}\right\|_{3 / 2} & \leq\left\|\nabla\left(\varphi_{1}-\varphi_{2}\right)\right\|_{2}\left\|\mathbf{B}_{1}\right\|_{6}+\left\|\nabla \varphi_{2}\right\|_{2}\left\|\mathbf{B}_{1}-\mathbf{B}_{2}\right\|_{6} \\
& \leq c_{0}\left(\left\|\mathbf{B}_{1}\right\|_{\mathbf{H}^{1}}\left\|\varphi_{1}-\varphi_{2}\right\|_{\mathscr{H}^{1}}+\left\|\varphi_{2}\right\|_{\mathscr{H}^{1}}\left\|\mathbf{B}_{1}-\mathbf{B}_{2}\right\|_{\mathbf{H}^{1}}\right),
\end{aligned}
$$

where $c_{0}$ is a positive constant related to the continuous imbedding of $H^{1}(\Omega)$ in the space $L^{6}(\Omega),\left(\Omega \subset \mathbb{R}^{3}\right)$. Hence

$$
\left\|\nabla \varphi_{1} \cdot \mathbf{B}_{1}-\nabla \varphi_{2} \cdot \mathbf{B}_{2}\right\|_{3 / 2} \leq c_{0} R\left\|v_{1}-v_{2}\right\|_{\mathbb{Q}^{1} 1} .
$$

For the second term, we have

$$
\begin{aligned}
\left\|\varphi_{1}\left|\mathbf{B}_{1}+\mathbf{A}_{\mathbf{H}}\right|^{2}-\varphi_{2}\left|\mathbf{B}_{2}+\mathbf{A}_{\mathbf{H}}\right|^{2}\right\|_{3 / 2} \\
\leq\left\|\left.\left(\varphi_{1}-\varphi_{2}\right)\left|\mathbf{B}_{1}+\mathbf{A}_{\mathbf{H}}\right|^{2}\right|_{3 / 2}+\right\| \varphi_{2}\left(\mathbf{B}_{1}-\mathbf{B}_{2}\right)\left(\mathbf{B}_{1}+\mathbf{B}_{2}+2 \mathbf{A}_{\mathbf{H}}\right) \|_{3 / 2} \\
\leq\left\|\varphi_{1}-\varphi_{2}\right\|_{3}\left\|\mathbf{B}_{1}+\mathbf{A}_{\mathbf{H}}\right\|_{6}^{2}+\left\|\varphi_{2}\right\|_{3}\left\|\mathbf{B}_{1}-\mathbf{B}_{2}\right\|_{6}\left\|\mathbf{B}_{1}+\mathbf{B}_{2}+2 \mathbf{A}_{\mathbf{H}}\right\|_{6} \\
\leq c_{0}^{3}\left[2\left(\left\|\mathbf{B}_{1}\right\|_{\mathbf{H}^{1}}^{2}+\left\|\mathbf{A}_{\mathbf{H}}\right\|_{\mathbf{H}^{1}}^{2}\right)\left\|\varphi_{1}-\varphi_{2}\right\| \mathscr{H}^{1}\right. \\
\left.\quad+\left\|\varphi_{2}\right\|_{\mathscr{H}^{1}}\left(\left\|\mathbf{B}_{1}\right\|_{\mathbf{H}^{1}}+\left\|\mathbf{B}_{2}\right\|_{\mathbf{H}^{1}}+2\left\|\mathbf{A}_{\mathbf{H}}\right\|_{\mathbf{H}^{1}}\right)\left\|\mathbf{B}_{1}-\mathbf{B}_{2}\right\|_{\mathbf{H}^{1}}\right]
\end{aligned}
$$


Since $\mathbf{H} \in L^{\infty}\left(0, T ; \mathbf{L}^{2}(\Omega)\right)$, there is a constant $c_{1}>0$ independent from $t$ such that $\left\|\mathbf{A}_{\mathbf{H}}(t)\right\|_{\mathbf{H}^{1}} \leq c_{1}$ in $[0, T]$. Then it follows

$$
\left\|\varphi_{1}\left|\mathbf{B}_{1}+\mathbf{A}_{\mathbf{H}}\right|^{2}-\varphi_{2}\left|\mathbf{B}_{2}+\mathbf{A}_{\mathbf{H}}\right|^{2}\right\|_{3 / 2} \leq c_{0}^{3}\left(2\left(R^{2}+c_{1}^{2}\right)+2 R\left(R+c_{1}\right)\right)\left\|v_{1}-v_{2}\right\|_{\mathbb{R}^{1}} .
$$

The other terms are estimated analogously. Therefore, we obtain a constant $C_{R}$ independent from $t$ and depending on $R, T$ and $\mathbf{H}$ such that for each $t \in[0, T]$

$$
\left\|\mathscr{F}^{\prime}\left(t, v_{1}\right)-\mathscr{F}^{\prime}\left(t, v_{2}\right)\right\|_{3 / 2} \leq C_{R}\left\|v_{1}-v_{2}\right\|_{\boxplus 11} \quad \forall\left(v_{1}, v_{2}\right) \in B_{R}^{2}
$$

On the other hand, for $t_{1}, t_{2} \in[\tau, T]\left(\tau>0\right.$ arbitrary) and $v=(\varphi, \mathbf{B})$ in $B_{R}$, we have

$$
\begin{aligned}
\left\|\mathscr{F}^{\prime}\left(t_{1}, v\right)-\mathscr{F}^{\prime}\left(t_{2}, v\right)\right\|_{3 / 2} \leq & \frac{2}{\eta \kappa}\left\|\nabla \varphi\left(\mathbf{A}_{\mathbf{H}}\left(t_{1}\right)-\mathbf{A}_{\mathbf{H}}\left(t_{2}\right)\right)\right\|_{3 / 2} \\
& +\frac{1}{\eta}\left\|\varphi\left(\left|\mathbf{B}+\mathbf{A}_{\mathbf{H}}\left(t_{1}\right)\right|^{2}-\left|\mathbf{B}+\mathbf{A}_{\mathbf{H}}\left(t_{2}\right)\right|^{2}\right)\right\|_{3 / 2} \\
& +\left\||\varphi|^{2}\left(\mathbf{A}_{\mathbf{H}}\left(t_{1}\right)-\mathbf{A}_{\mathbf{H}}\left(t_{2}\right)\right)\right\|_{3 / 2} .
\end{aligned}
$$

As above, we estimate each term separately. For the first one, we obtain

$$
\begin{aligned}
\left\|\nabla \varphi\left(\mathbf{A}_{\mathbf{H}}\left(t_{1}\right)-\mathbf{A}_{\mathbf{H}}\left(t_{2}\right)\right)\right\|_{3 / 2} & \leq c_{0}\|\nabla \varphi\|_{2}\left\|\mathbf{A}_{\mathbf{H}}\left(t_{1}\right)-\mathbf{A}_{\mathbf{H}}\left(t_{2}\right)\right\|_{\mathbf{H}^{1}} \\
& \leq c_{0}\|\nabla \varphi\|_{2}\left\|\int_{t_{1}}^{t_{2}} \frac{\partial \mathbf{A}_{\mathbf{H}}}{\partial t}(t) \mathrm{d} t\right\|_{\mathbf{H}^{1}} \\
& \leq c_{0} R\left|t_{1}-t_{2}\right|^{1 / 2}\left(\int_{\tau}^{T}\left\|\frac{\partial \mathbf{A}_{\mathbf{H}}}{\partial t}(t)\right\|_{\mathbf{H}^{1}}^{2} \mathrm{~d} t\right)^{1 / 2} .
\end{aligned}
$$

The other remaining terms are estimated similarly. Therefore, we obtain that the mapping $\mathscr{F}^{\prime}$ is locally Hölder continuous in $\mathrm{t}$ and locally Lipschitz continuous in $v$. Here the sense of the definition is borrowed from [10]. We then conclude by applying the existence theorem in [10] (cf. Theorem 3.3.3) that for each $v_{0} \in \mathscr{D}\left(\mathscr{A}^{1 / 2}\right)$ the initial value problem (3.14) has a unique local strong solution $v=\left(\psi, \mathbf{A}^{\prime}\right)$ on an interval $\left[0, T_{1}\right)$ with $0<T_{1} \leq T$. More precisely, $v \in C\left(\left[0, T_{1}\right), \mathbb{W}^{1}(\Omega)\right) \cap C^{1}\left(\left(0, T_{1}\right), \mathbb{W}^{1}(\Omega)\right)$ and

$$
\frac{\mathrm{d} v}{\mathrm{~d} t}+\mathscr{B} v(t)=\mathscr{F}^{\prime}(t, v(t)) \quad \text { for a.e } 0<t<T_{1}, v(0)=v_{0}
$$

The proof of existence of the solution $v$ on the entire interval $[0, T]$ uses an a-priori bound on the energy-type functional associated to the initial value problem (3.14)

$$
\begin{aligned}
E_{\omega}(t, \varphi, \mathbf{B})= & \int_{\Omega}\left[\left|\left(\frac{i}{\kappa} \nabla+\mathbf{B}\right) \varphi\right|^{2}+\frac{1}{2}\left(1-|\varphi|^{2}\right)^{2}+2 \omega(\nabla \cdot \mathbf{B})^{2}+|\nabla \times \mathbf{B}-\mathbf{H}|^{2}\right] \mathrm{d} x \\
& +\frac{1}{\kappa^{2}} \int_{\partial \Omega} \gamma|\varphi|^{2} \mathrm{~d} \sigma(x)
\end{aligned}
$$


defined for $t \geq 0$ and $(\varphi, \mathbf{B}) \in \mathbb{U}^{1}(\Omega)$. We set $E_{\omega}(t)=E_{\omega}(t, \psi(t), \mathbf{A}(t))$ with $\mathbf{A}=\mathbf{A}^{\prime}+\mathbf{A}_{\mathbf{H}}$. We have for each fixed $t, \mathbf{A}_{\mathbf{H}}$ is a weak solution for the boundary value problem (2.1)(2.2). It follows that for all $t \in\left(0, T_{1}\right)$, the time derivative of $E_{\omega}$ takes the following expression:

$$
\begin{aligned}
\frac{\mathrm{d} E_{\omega}}{\mathrm{d} t}(t)= & -2 \int_{\Omega}\left[\eta\left|\frac{\partial \psi}{\partial t}-i \omega \kappa \psi(\nabla \cdot \mathbf{A})\right|^{2}+\left|\frac{\partial \mathbf{A}}{\partial t}\right|^{2}+\omega^{2}|\nabla(\nabla \cdot \mathbf{A})|^{2}\right] \mathrm{d} x \\
& -2 \int_{\Omega} \frac{\partial \mathbf{A}_{\mathbf{H}}}{\partial t} \cdot \frac{\partial \mathbf{A}}{\partial t} \mathrm{~d} x-2 \int_{\Omega} \frac{\partial \mathbf{H}}{\partial t} \cdot(\nabla \times \mathbf{A}-\mathbf{H}) \mathrm{d} x,
\end{aligned}
$$

which together with Hölder inequality yields

$$
\begin{aligned}
\frac{\mathrm{d} E_{\omega}}{\mathrm{d} t}(t) & \leq\left\|\frac{\partial \mathbf{A}_{\mathbf{H}}}{\partial t}\right\|_{2}^{2}+\|\nabla \times \mathbf{A}-\mathbf{H}\|_{2}^{2}+\left\|\frac{\partial \mathbf{H}}{\partial t}\right\|_{2}^{2} \\
& \leq E_{\omega}(t)+\left\|\frac{\partial \mathbf{A}_{\mathbf{H}}}{\partial t}\right\|_{2}^{2}+\left\|\frac{\partial \mathbf{H}}{\partial t}\right\|_{2}^{2}
\end{aligned}
$$

Using standard arguments, we infer for all $0<\tau \leq t<T_{1}$

$$
E_{\omega}(t) \leq \mathrm{e}^{(t-\tau)} E_{\omega}(\tau)+\int_{\tau}^{t} \mathrm{e}^{(t-s)}\left(\left\|\frac{\partial \mathbf{H}}{\partial t}(s)\right\|_{2}^{2}+\left\|\frac{\partial \mathbf{A}_{\mathbf{H}}}{\partial t}(s)\right\|_{2}^{2}\right) \mathrm{d} s .
$$

The assumption $\mathbf{H} \in W^{1,2}\left(\tau, T ; \mathbf{L}^{2}(\Omega)\right)$ provides then a bound on the solution $v$ of problem (3.14) on the interval $\left[\tau, T_{1}\right)(\tau>0$ is sufficiently small). Moreover, $v \in C([0, \tau]$, $\left.\mathbb{M}^{1}(\Omega)\right)$. It follows that the solution $v$ is bounded in the interval $\left[0, T_{1}\right)$. Therefore, the maximal interval of existence of $v$ coincides with $[0, T)$. On the other hand by applying Lemma 3.1 and according to the continuity of the map $\left(t \geq 0 \rightarrow \mathrm{e}^{-\mathscr{B} t} v_{0} \in \mathbb{H}^{1}(\Omega)\right)$, we obtain that the solution $v$ is continuous at $t=T$. Finally, since $\left(t \rightarrow \mathrm{e}^{-\mathscr{B} t} v_{0}\right) \in C^{1}((0, T]$, $\left.\mathbb{\square}^{1}(\Omega)\right)$ and once again by using Lemma 3.1, we conclude

$$
v \in C^{\theta}\left([\tau, T], \mathbb{H}^{1}(\Omega)\right) \quad \forall 0<\tau \leq T, 0<\theta<\frac{1}{4} .
$$

This concludes the proof of Theorem 3.2.

We remark that Theorem 3.2 implies existence of strong solutions to the TDGL equations in the case when the applied magnetic field $\mathbf{H}$ is time independent.

Corollary 3.3. If the magnetic field $\mathbf{H}$ is time independent then for each initial data $u_{0}$ in $\mathscr{D}\left(\mathscr{A}^{1 / 2}\right)$, the problem (2.16) has a unique strong solution $v=\left(\psi, \mathbf{A}^{\prime}\right) \in C^{\theta}\left([\tau, T], \mathbb{H}^{1}(\Omega)\right)$ for all $0<\tau \leq T$ and $0<\theta<1 / 4$.

We consider now the remaining integral part in equation $(2.17)$ introduced by $\partial \mathbf{H} / \partial t$

$$
\mathscr{E}_{\mathbf{H}}(t)=\int_{0}^{t} \mathrm{e}^{-\mathscr{E}(t-s)} \frac{\partial \mathbf{A}_{\mathbf{H}}}{\partial t}(s) \mathrm{d} s
$$

where $\mathscr{E}$ is the restriction of the operator $\mathscr{A}$ to $\mathrm{L}^{2}(\Omega)$. Obviously, a necessary condition for existence of mild solutions to the problem (2.16) is the continuity in time of $\mathscr{E}_{\mathbf{H}}$. In 
other words, we need to prove that the following initial value problem

$$
\left\{\begin{array}{l}
\frac{\mathrm{d} w}{\mathrm{~d} t}+\mathscr{E} w=\frac{\partial \mathrm{A}_{\mathrm{H}}}{\partial t}(t) \text { for } t>0 \\
w(0)=0
\end{array}\right.
$$

defined in $\mathbf{L}^{2}(\Omega)$ has as mild solution $w=\mathscr{F}_{\mathbf{H}}$.

Lemma 3.4. We suppose $\mathbf{H}$ satisfies (3.1) and (3.2). Then $\mathscr{F}_{\mathbf{H}} \in C\left([0, T], \mathbf{H}^{1}(\Omega)\right)$.

Proof. As mentioned before, only the case $1 / 2 \leq \mu<1$ remains to be treated. We set $w(t)=$ $\mathscr{F}_{\mathbf{H}}(t)$. First we show that for all $t \in[0, T], w(t)$ is well defined and belongs to $\mathbf{H}^{1}(\Omega)$. Indeed, since $\left(\mathrm{e}^{-\mathscr{E} t}\right)_{t \geq 0}$ is in particular a $C_{0}$-semigroup in $\mathbf{H}^{1}(\Omega)$, there is a constant $M$ depending on $T$ such that

$$
\|w(t)\|_{\mathbf{H}^{1}} \leq M \int_{0}^{t}\left\|\frac{\partial \mathbf{A}_{\mathbf{H}}}{\partial t}(s)\right\|_{\mathbf{H}^{1}} \mathrm{~d} s .
$$

Let $t \in(0, T]$ and $\varepsilon>0$. According to (3.2), there is $\alpha>0$ small enough such that

$$
\left\|\frac{\partial \mathbf{A}_{\mathbf{H}}}{\partial t}(t)\right\|_{\mathbf{H}^{1}} \leq \varepsilon t^{-\mu} \quad \forall 0<t \leq \alpha .
$$

For $t>\alpha$ it follows that

$$
\|w(t)\|_{\mathbf{H}^{1}} \leq M\left(\varepsilon \int_{0}^{\alpha} s^{-\mu} \mathrm{d} s+\left\|\frac{\partial \mathbf{A}_{\mathbf{H}}}{\partial t}\right\|_{L^{1}\left(\alpha, T ; \mathbf{H}^{1}(\Omega)\right)}\right) .
$$

Therefore $\|w(t)\|_{\mathbf{H}^{1}}<\infty$ for all $t \in(0, T]$. Moreover (3.36) and (3.37) imply

$$
\lim _{t \rightarrow 0^{+}}\|w(t)\|_{\mathbf{H}^{1}}=0
$$

Let now $h \geq 0$ and $t \in(0, T)$. We write

$$
\begin{gathered}
w(t+h)-w(t)=I_{1}+I_{2}, \\
I_{1}=\int_{0}^{t}\left(\mathrm{e}^{-\mathscr{E} h}-I\right) \mathrm{e}^{-\mathscr{E}(t-s)} \frac{\partial \mathbf{A}_{\mathbf{H}}}{\partial t}(s) \mathrm{d} s, \\
I_{2}=\int_{t}^{t+h} \mathrm{e}^{-\mathscr{E}(t+h-s)} \frac{\partial \mathbf{A}_{\mathbf{H}}}{\partial t}(s) \mathrm{d} s .
\end{gathered}
$$

On the other hand, the semigroup $\left(\mathrm{e}^{-\mathscr{E} t}\right)_{t \geq 0}$ satisfies

$$
\begin{gathered}
\left\|\mathscr{E}^{\alpha} \mathrm{e}^{-\mathscr{E} t} \mathbf{B}\right\|_{2} \leq c_{\alpha} t^{-\alpha} \mathrm{e}^{-\delta t}\|\mathbf{B}\|_{2} \quad \forall t>0, \alpha \geq 0, \mathbf{B} \in \mathbf{L}^{2}(\Omega), \\
\left\|\left(\mathrm{e}^{-\mathscr{E} t}-I\right) \mathbf{B}\right\|_{2} \leq c_{\alpha}^{\prime} t^{\alpha}\left\|\mathscr{E}^{\alpha} \mathbf{B}\right\|_{2} \quad \forall t \geq 0,0<\alpha \leq 1, \mathbf{B} \in \mathbf{B} \in \mathscr{D}\left(\mathscr{E}^{\alpha}\right) .
\end{gathered}
$$


874 On strong solutions of the Ginzburg-Landau equations

First we have

$$
\left\|I_{1}\right\|_{\mathbf{H}^{1}} \leq \int_{0}^{t}\left\|\left(\mathrm{e}^{-\mathscr{E} h}-I\right) \mathrm{e}^{-\mathscr{E}(t-s)}\left(\mathscr{E} 1 / 2 \frac{\partial \mathbf{A}_{\mathbf{H}}}{\partial t}(s)\right)\right\|_{2} \mathrm{~d} s,
$$

which by using (3.41) and (3.42) gives

$$
\left\|I_{1}\right\|_{\mathbf{H}^{1}} \leq C h^{\theta} \int_{0}^{t}(t-s)^{-\theta}\left\|\frac{\partial \mathbf{A}_{\mathbf{H}}}{\partial t}(s)\right\|_{\mathbf{H}^{1}} \mathrm{~d} s,
$$

with $0<\theta<1$ arbitrary and $C$ a constant independent from $t$ and $h$.

Let $0<\theta<1-\mu$ and fix $p \in(1,2)$ such that

$$
p \mu<1, \quad 0<\theta<\frac{p-1}{p} .
$$

We distinguish two cases. If $t \leq \alpha,(3.37)$ and Hölder inequality then imply

$$
\begin{aligned}
\left\|I_{1}\right\|_{\mathbf{H}^{1}} & \leq C h^{\theta} \varepsilon \int_{0}^{\alpha}(t-s)^{-\theta} s^{-\mu} \mathrm{d} s \\
& \leq C h^{\theta} \varepsilon\left(\int_{0}^{\alpha}(t-s)^{-\theta(p / p-1)} \mathrm{d} s\right)^{p-1 / p}\left(\int_{0}^{\alpha} s^{-p \mu} \mathrm{d} s\right)^{1 / p} .
\end{aligned}
$$

If $t>\alpha$, we obtain

$$
\begin{aligned}
\left\|I_{1}\right\|_{\mathbf{H}^{1}} & \leq C h^{\theta}\left(\varepsilon \int_{0}^{\alpha / 2}(t-s)^{-\theta} s^{-\mu} \mathrm{d} s+\int_{\alpha / 2}^{t}(t-s)^{-\theta}\left\|\frac{\partial \mathbf{A}_{\mathbf{H}}}{\partial t}(s)\right\|_{\mathbf{H}^{1}} \mathrm{~d} s\right) \\
& \leq C h^{\theta}\left[\varepsilon(\alpha / 2)^{-\theta} \int_{0}^{\alpha / 2} s^{-\mu} \mathrm{d} s+\left(\int_{\alpha / 2}^{t}(t-s)^{-2 \theta} \mathrm{d} s\right)^{1 / 2}\left\|\frac{\partial \mathbf{A}_{\mathbf{H}}}{\partial t}\right\|_{L^{2}\left(\alpha / 2, T ; \mathbf{H}^{1}(\Omega)\right)}\right] .
\end{aligned}
$$

On the other hand, as in (3.36) we get

$$
\left\|I_{2}\right\|_{\mathbf{H}^{1}} \leq M \int_{t}^{t+h}\left\|\frac{\partial \mathbf{A}_{\mathbf{H}}}{\partial t}(s)\right\|_{\mathbf{H}^{1}} \mathrm{~d} s .
$$

First if $t+h \leq \alpha$, then from (3.37) we obtain

$$
\left\|I_{2}\right\|_{\mathbf{H}^{1}} \leq M \varepsilon \int_{t}^{t+h} s^{-\mu} \mathrm{d} s \leq M \varepsilon h^{(p-1) / p}\left(\int_{0}^{T} s^{-p \mu} \mathrm{d} s\right)^{1 / p} .
$$

Second if $t \geq \alpha$, then

$$
\left\|I_{2}\right\|_{\mathbf{H}^{1}} \leq M h^{1 / 2}\left\|\frac{\partial \mathbf{A}_{\mathbf{H}}}{\partial t}\right\|_{L^{2}\left(\alpha, T ; \mathbf{H}^{1}(\Omega)\right)} .
$$


Finally if $t \leq \alpha \leq t+h$, then

$$
\begin{aligned}
\left\|I_{2}\right\|_{\mathbf{H}^{1}} & \leq M\left(\varepsilon \int_{t}^{\alpha} s^{-\mu} \mathrm{d} s+\int_{\alpha}^{t+h}\left\|\frac{\partial \mathbf{A}_{\mathbf{H}}}{\partial t}(s)\right\|_{\mathbf{H}^{1}(\Omega)} \mathrm{d} s\right) \\
& \leq M\left[\varepsilon(\alpha-t)^{(p-1) / p}\left(\int_{0}^{T} s^{-p \mu} \mathrm{d} s\right)^{1 / p}+(t+h-\alpha)^{1 / 2}\left\|\frac{\partial \mathbf{A}_{\mathbf{H}}}{\partial t}\right\|_{L^{2}\left(\alpha, T ; \mathbf{H}^{1}(\Omega)\right)}\right] \\
& \leq M\left[\varepsilon h^{(p-1) / p}\left(\int_{0}^{T} s^{-p \mu} \mathrm{d} s\right)^{1 / p}+h^{1 / 2}\left\|\frac{\partial \mathbf{A}_{\mathbf{H}}}{\partial t}\right\|_{L^{2}\left(\alpha, T ; \mathbf{H}^{1}(\Omega)\right)}\right] .
\end{aligned}
$$

The cases $t=0$ and $t=T$ follow similarly. Therefore we obtain

$$
w \in C^{\theta}\left([0, T], \mathbf{H}^{1}(\Omega)\right) \quad \forall \theta \in(0,1-\mu) .
$$

Moreover, since $1 / 2 \leq \mu<1$, we have $w \in C^{\theta}\left([0, T], \mathbf{H}^{1}(\Omega)\right)$ for all $0<\theta<1 / 2$. In particular $w \in C\left([0, T], \mathbf{H}^{1}(\Omega)\right)$, which concludes the proof of Lemma 3.4.

Remark 3.5. Concerning the case $\mu<1 / 2$, one can prove likewise the Hölder continuity in time of $\mathscr{F}_{\mathbf{H}}$. More precisely, we have for all $\mu<1$

$$
\mathscr{F}_{\mathbf{H}} \in C^{\theta}\left([0, T], \mathbf{H}^{1}(\Omega)\right) \quad \forall \theta \in\left(0, \frac{1}{2}\right) .
$$

Following Theorem 3.2 and Lemma 3.4, a result concerning the mild solutions of the initial value problem (2.16) can be derived.

Theorem 3.6. We suppose the magnetic field $\mathbf{H}$ satisfies assumptions (3.1) and (3.2). Then for each initial data $u_{0} \in \mathscr{D}\left(\mathscr{A}^{1 / 2}\right)$ the problem (2.16) possesses a unique mild solution $u=$ $\left(\psi, \mathbf{A}^{\prime}\right) \in C^{\theta}\left([\tau, T], \mathbb{H}^{1}(\Omega)\right)$ for all $0<\tau \leq T$ and $0<\theta<1 / 4$.

Proof. The proof of local existence and uniqueness is based on the contraction mapping principle. Let the initial data $u_{0}=\left(\psi_{0}, \mathbf{A}_{0}^{\prime}=\mathbf{A}_{0}-\mathbf{A}_{\mathbf{H}}(0)\right) \in \mathscr{D}\left(\mathscr{A}^{1 / 2}\right)$, we define for all $v=$ $(\varphi, \mathbf{B}) \in L^{\infty}\left(0, T ; \mathbb{H}^{1}(\Omega)\right)$

$$
\mathscr{G}(v)(t)=\mathrm{e}^{-\mathscr{A} t} u_{0}+\int_{0}^{t} \mathrm{e}^{-\mathscr{B}(t-s)} \mathscr{F}(s, v(s)) \mathrm{d} s \quad \text { for } 0 \leq t \leq T .
$$

By setting

$$
\mathscr{G}_{1}(v)(t)=\mathrm{e}^{-\mathscr{A} t} u_{0}+\int_{0}^{t} \mathrm{e}^{-\mathscr{B}(t-s)} \mathscr{F}^{\prime}(s, v(s)) \mathrm{d} s \quad \text { for } 0 \leq t \leq T .
$$

We have $\mathscr{G}(v)(t)=\mathscr{G}_{1}(v)(t)-\left(0, \mathscr{I}_{\mathbf{H}}(t)\right)$. Let now $B_{R}\left(u_{0}\right)$ be the ball in $\mathbb{H}^{1}(\Omega)$ with radius $R>0$ arbitrary and centered at $u_{0}$. For $\tau>0$ small enough, we define

$$
\mathscr{X}=\left\{v \in C\left([0, \tau], \mathbb{H}^{1}(\Omega)\right): v(t) \in B_{R}\left(u_{0}\right) \forall t \in[0, \tau]\right\},
$$


endowed with the norm

$$
\|v\|_{\mathscr{Q}}=\sup _{0 \leq t \leq \tau}\|v(t)\|_{\mathbb{R}^{1}}
$$

Obviously, $\mathscr{X}$ is a closed convex subset of the Banach space $C\left([0, \tau], \mathbb{H}^{1}(\Omega)\right)$. We must show that $\mathscr{G}$ maps $\mathscr{X}$ in itself and is a contraction mapping on $\mathscr{X}$. Indeed, according to the proof of Theorem 3.2, the mapping $\mathscr{G}_{1}$ maps $\mathscr{X}$ in itself for some $\tau>0$ sufficiently small and acts as a contraction, see [10, Theorem 3.3.3]. On the other hand, by virtue of Lemma 3.4, we have $\mathscr{E}_{\mathbf{H}} \in C\left([0, T], \mathbb{H}^{1}(\Omega)\right)$. It follows that for some $\tau>0$ small enough, the mapping $\mathscr{G}$ maps $\mathscr{X}$ in itself and is a contraction mapping on $\mathscr{X}$. Therefore, the mapping $\mathscr{G}$ possess a unique fixed point $u=\left(\psi, \mathbf{A}^{\prime}\right) \in \mathscr{X}$ (see [24]), that is,

$$
u(t)=\mathrm{e}^{-\mathscr{A} t} u_{0}+\int_{0}^{t} \mathrm{e}^{-\mathscr{P}(t-s)} \mathscr{F}(s, u(s)) \mathrm{d} s \quad \text { for } 0 \leq t \leq \tau .
$$

The proof of existence of the solution $u$ on the entire interval $[0, T]$ uses an a-priori bound on the energy-type functional $E_{\omega}$ defined in (3.29). We put $E_{\omega}(t)=E_{\omega}(t, \psi(t), \mathbf{A}(t))$ where $\mathbf{A}=\mathbf{A}^{\prime}+\mathbf{A}_{\mathbf{H}}$ as defined in (2.3). $\mathscr{F}_{\mathbf{H}}$ being bounded in $[0, T]$, we compute the time derivative of $E_{\omega}(t)$ and argue similarly as in Theorem 3.2. It follows the solution $u=\left(\psi, \mathbf{A}^{\prime}\right)$ is bounded in the entire interval $[0, T]$. Therefore the maximal interval of existence of the mild solution coincides with $[0, T]$. Furthermore, by virtue of Theorem 3.2, Lemma 3.4, and Remark 3.5, we obtain that $u \in C\left([0, T], \mathbb{H}^{1}(\Omega)\right) \cap C^{\theta}\left([\tau, T], \mathbb{M}^{1}(\Omega)\right)$ for all $0<\tau \leq$ $T$ and $0<\theta<1 / 4$, which concludes the proof of Theorem 3.6.

Remark 3.7. Set $v(t)=u(t)+\left(0, \mathscr{I}_{\mathbf{H}}(t)\right)$. By estimating the energy functional $E_{\omega}$, we obtain that $\mathrm{d} v / \mathrm{d} t \in L^{2}\left(\tau, T ; \mathbb{L}^{2}(\Omega)\right)$ for all $0<\tau \leq T$. Moreover by virtue of Theorem 3.2, we have $v(t) \in \mathscr{D}(\mathscr{B})$ a.e $t \in(0, T)$ and

$$
\frac{\mathrm{d} v}{\mathrm{~d} t}+\mathscr{B} v(t)=\mathscr{F}^{\prime}(t, u(t)) \quad \text { for a.e } 0<t<T .
$$

On the other hand, we have that $\mathscr{E}$ is a positive, selfadjoint linear operator with compact resolvent on the Hilbert space $\mathbf{L}^{2}(\Omega)$ and $\mathrm{d} \mathbf{A}_{\mathbf{H}} / \mathrm{d} t \in L_{l o c}^{2}\left(0, T ; \mathscr{D}\left(\mathscr{E}^{1 / 2}\right)\right)$. Therefore we can use some general properties on the regularity of the solutions of evolutionary equations to derive that $\mathscr{F}_{\mathbf{H}}$ is in fact a strong solution of equation (3.36) in the space $\mathbf{H}^{1}(\Omega)$ and on the interval $(0, T)$. Moreover, we have

$$
\begin{aligned}
& \frac{\mathrm{d} \mathscr{L}_{\mathbf{H}}}{\mathrm{d} t} \in L^{2}\left(\tau, T ; \mathbf{L}^{2}(\Omega)\right) \quad \text { for } 0<\tau<T, \\
& \mathscr{F}_{\mathbf{H}} \in C\left([0, T] ; \mathbf{H}^{k}(\Omega)\right) \quad \text { for } 0 \leq k<1 \text {. }
\end{aligned}
$$

One may consult [16, Theorem 42.12 and Corollary 42.13]. Concerning the divergence of the vector potential A, using once again the energy functional, we obtain

$$
\nabla \cdot \mathbf{A} \in L^{2}\left(\tau, T ; H^{1}(\Omega)\right) \quad \forall 0<\tau \leq T .
$$

As a consequence of Remark 3.7, we have the following. 
Corollary 3.8. The mild solutions of problem (2.16) obtained in Theorem 3.6 are in fact strong solutions, that is, $u(t) \in \mathscr{D}(\mathscr{B})$ a.e $t \in(0, T), \mathrm{d} u / \mathrm{d} t \in L^{2}\left(\tau, T ; \mathbb{L}^{2}(\Omega)\right)$ for $0<\tau<T$ and

$$
\frac{\mathrm{d} u}{\mathrm{~d} t}+\mathscr{B} u(t)=\mathscr{F}(t, u(t)) \quad \text { for a.e } 0<t<T \text {. }
$$

Furthermore, if $\mathbf{H} \in W^{1,2}\left(0, T ; \mathbf{L}^{2}(\Omega)\right)$, we obtain that $\mathrm{d} u / \mathrm{d} t \in L^{2}\left(0, T ; \mathbb{L}^{2}(\Omega)\right)$.

Remark 3.9. Since $\mathbf{A}_{\mathbf{H}}$ is a weak solution for equations (2.1)-(2.2), then the vector potential A satisfies the TDGL equations only in a weak sense. However, if we assume $\mathbf{H}(t) \in \mathbf{H}^{1}(\Omega)$ for all $t>0$, then we obtain that the pair $(\psi, \mathbf{A})$ is a strong solution of the system of equations (1.6)-(1.9).

Remark 3.10. It is not hard to see that the order parameter $\psi$ satisfies the maximum modulus principle. More precisely, if at instant $t=t_{0} \in[0, T]$ we assume that

$$
\left\|\psi\left(t_{0}\right)\right\|_{\infty} \leq C
$$

Then we obtain for all subsequent instant $t$

$$
\|\psi(t)\|_{\infty} \leq \max (1, C) \quad \forall t \in\left[t_{0}, T\right] .
$$

However, for the case of steady solutions, it is known that always $|\psi(x)| \leq 1$ for all $x \in \Omega$. For details about the proofs, we refer to $[11,18]$.

\section{Large-time asymptotic behavior}

This section is concerned with the asymptotic behavior of solutions of the TDGL equations. In [6], the authors show that the mild solutions $u=\left(\psi, \mathbf{A}^{\prime}\right)$ define a dynamical process in a chosen Hilbert space, namely $\mathscr{D}\left(\mathscr{A}^{\alpha}\right)$ for $3 / 4<\alpha<1$. This process completely describes the dynamics of the TDGL equations. Our aim here is first to extend this process to $\mathscr{D}\left(\mathscr{A}^{1 / 2}\right)$ and second to investigate the asymptotic behavior of the strong solutions in the case where the magnetic field $\mathbf{H}$ is stationary. Throughout the section, the sense of definitions and notion appearing is borrowed from $[9,20]$.

THEOREM 4.1. The strong solutions obtained in Theorem 3.6 generate a dynamical process $\mathcal{U}=\{U(t, s): 0 \leq s \leq t \leq T\}$ on $\mathscr{D}\left(\mathscr{A}^{1 / 2}\right)$ by the definition

$$
u(t)=U(t, s) u(s) \quad \forall 0 \leq s \leq t \leq T .
$$

Furthermore, for $(t, s)$ fixed with $s<t$, the map $U(t, s): \mathscr{D}\left(\mathscr{A}^{1 / 2}\right) \rightarrow \mathscr{D}\left(\mathscr{A}^{1 / 2}\right)$ is completely continuous, that is, it maps bounded sets into relatively compact sets.

Proof. Let $w_{s} \in \mathscr{D}\left(\mathscr{A}^{1 / 2}\right)$ and $0 \leq s \leq t \leq T$. We define $w(t):=U(t, s) w_{s}$ to be the mild solution of the following initial value problem

$$
\left\{\begin{array}{l}
\frac{\mathrm{d} w}{\mathrm{~d} t}+\mathscr{B} w=\mathscr{F}(t, w(t)) \quad \text { for } t>s \\
w(s)=w_{s} .
\end{array}\right.
$$


In other words

$$
w(t)=U(t, s) w_{s}=\mathrm{e}^{-\mathscr{B}(t-s)} w_{s}+\int_{s}^{t} \mathrm{e}^{-\mathscr{B}(t-r)} \mathscr{F}(r, w(r)) \mathrm{d} r .
$$

As we have proceeded previously, we have that $w \in C\left([s, T], \mathbf{H}^{1}(\Omega)\right)$ and $w$ is a strong solution for problem (4.2). The continuity of $U(t, s) w_{s}$ with respect to $t, s$ and $w_{s}$ follows from similar arguments. Furthermore, we have that $U(t, t)$ is equal to the identity and the uniqueness of the strong solutions implies

$$
U(t, s) U(s, r)=U(t, r) \quad \text { for } 0 \leq r \leq s \leq t \leq T .
$$

In particular, we obtain for $r=0$

$$
U(t, s) u(s)=u(t) \quad \text { for } 0 \leq s \leq t \leq T .
$$

The complete continuity of $U(t, s)$ is a consequence of the compact imbedding of $\mathscr{D}\left(\mathscr{B}^{\gamma}\right)$ in $\mathbb{H}^{1}(\Omega)$ for $3 / 4<\gamma<1$. Indeed, if $U$ is any bounded subset of $\mathscr{D}\left(\mathscr{A}^{1 / 2}\right)$, then using standard arguments yields the boundedness of $U(t, s) \mathcal{U}$ in $\mathscr{D}\left(\mathscr{B}^{\gamma}\right)$ with $3 / 4<\gamma<1$. This concludes the proof of Theorem 4.1.

Remark 4.2. If the magnetic field $\mathbf{H}$ is independent from the time, then the nonlinearity $\mathscr{F}_{\mathrm{f}}$ becomes independent from $t$ and therefore the dynamical process becomes a dynamical system $\mathscr{S}=\{S(t): t \geq 0\}$ with

$$
S(t-s)=U(t, s) \quad \forall 0 \leq s \leq t
$$

Moreover, the functional $E_{\omega}$ given by (3.29) becomes in this case a Lyapunov functional for the dynamical system. The dynamical system $\mathscr{S}$ is also called a gradient system, see [9]. On the other hand, according to Theorem 4.1 we have that the orbit of each $u_{0}$ is relatively compact in $\mathscr{D}\left(\mathscr{A}^{1 / 2}\right)$. Concerning the omega-limit set $\omega\left(u_{0}\right)$ of each $u_{0}$, we may apply [9, cf. Lemma 3.1.1]. It follows that for all $u_{0} \in \mathscr{D}\left(\mathscr{A}^{1 / 2}\right), \omega\left(u_{0}\right)$ is a nonempty compact, invariant and connected set and

$$
\operatorname{dist}\left(S(t) u_{0}, \omega\left(u_{0}\right)\right) \longrightarrow 0 \quad \text { as } t \rightarrow \infty \text {. }
$$

Moreover, by applying a result in [10, cf., Theorem 4.3.4], we obtain

$$
S(t) u_{0} \longrightarrow M \quad \text { as } t \rightarrow \infty,
$$

where $M$ is a maximal invariant subset of the set $\left\{u \in \mathscr{D}\left(\mathscr{A}^{1 / 2}\right): \mathrm{d} / \mathrm{d} t\left[E_{\omega}(S(t) u)\right]=0\right\}$. This implies the fact that every solution is attracted to a set of divergence free equilibria. 
These consist of the pairs $\left(\psi, \mathbf{A}^{\prime}=\mathbf{A}-\mathbf{A}_{\mathbf{H}}\right)$, where $\psi$ and $\mathbf{A}$ are the solutions of the timeindependent Ginzburg-Landau equations

$$
\begin{gathered}
-\left(\frac{i}{\kappa} \nabla+\mathbf{A}\right)^{2} \psi+\left(1-|\psi|^{2}\right)=0 \quad \text { in } \Omega, \\
-\nabla \times \nabla \times \mathbf{A}+\mathbf{J}_{s}+\nabla \times \mathbf{H}=0 \quad \text { in } \Omega, \\
\mathbf{n} \cdot\left(\frac{i}{\kappa} \nabla+\mathbf{A}\right) \psi+\gamma \frac{i}{\kappa} \psi=0, \quad \mathbf{n} \times(\nabla \times \mathbf{A}-\mathbf{H})=0 \quad \text { on } \partial \Omega,
\end{gathered}
$$

in the London gauge " $\nabla \cdot \mathbf{A}=0$ ”. Solutions of (4.9)-(4.11) are also called steady solutions.

Theorem 4.3. The dynamical system $\mathscr{S}$ possesses a global attractor in the Hilbert space $\mathscr{D}\left(\mathscr{A}^{1 / 2}\right)$.

Proof. By virtue of [9, cf. Theorem 3.4.6], it suffices to verify the complete continuity of $S(t)$ for all $t>0$ and the point dissipativeness of the dynamical system $\mathscr{T}$, that is, the existence of a bounded set that attracts each point of $\mathscr{D}\left(\mathscr{A}^{1 / 2}\right)$. The first property follows from Theorem 4.1. Concerning the point dissipativeness, we point out the fact that the set of all steady solutions is bounded in $\mathbb{Q}^{1}(\Omega)$. We refer to [19, Lemma 5.2] for more details. Moreover, we have by virtue of Remark 4.2 that every solution is attracted to the set of steady solutions. Therefore $\{S(t): t \geq 0\}$ is point dissipative.

Remark 4.4. It is possible, according to [20], (cf. Theorem 4.1, Chapter VII), to characterize the global attractor in terms of the unstable manifold of the set $\mathscr{E}$ of equilibria, namely

$$
\mathscr{E}=\left\{w=(\psi, \mathbf{A}) \in \mathbb{H}^{1}(\Omega) \text { with }\left.\mathbf{n} \cdot \mathbf{A}\right|_{\partial \Omega}=0 \text { such that } S(t) w=w \forall t \geq 0\right\} .
$$

Moreover, if the set $\mathscr{E}$ is discrete, then the global attractor is the union of the heteroclinic curves joining one point of $\mathscr{E}$ with another point of $\mathscr{E}$. For details about these notions, one may consult [20]. Finally as in [12], when $\mathbf{H}$ is an asymptotically stationary field, that is, $\mathbf{H}$ approaches a stationary field as time goes to infinity, the dynamical process obtained in Theorem 4.1 is asymptotically autonomous. It means that the dynamical process asymptotically approaches a dynamical system; in addition, the attractors of both of them coincide.

\section{Uniform boundedness in time}

We investigate now to the question of boundedness for all times $t \geq 0$ of weak solutions $u=(\psi, \mathbf{A})$ of the gauged TDGL equations (1.6)-(1.9). In [6], the Lyapunov functional method ensures, in the case where $\mathbf{H} \in W^{1,2}\left(0, T ; L^{2}(\Omega)\right)$ for all $T>0$, global existence of weak solutions for all $t \geq 0$, and boundedness but only in each bounded interval $[0, T]$. It is the purpose here to look for conditions on $\mathbf{H}$ guaranteeing boundedness of the weak solutions with respect to all $t \geq 0$. In the sequel we consider the case where the order parameter satisfies at $t=0,\left\|\psi_{0}\right\|_{\infty} \leq 1$. According to Remark 3.10, we have $\|\psi(t)\|_{\infty} \leq 1$ for all $t \geq 0$. Furthermore, we assume that $\mathbf{H}$ satisfies (3.1) and (3.2). Without loss of generality we take $\omega=1$. Our main goal in this section is to improve the a-priori estimates on 
the solutions previously established in [22]. We recall that in [22], uniform boundedness in time of the solutions is proved by requiring $\mathbf{H} \in W^{1, \infty}\left(0, \infty ; \mathbf{L}^{2}(\Omega)\right)$. In the sequel, this assumption on $\mathbf{H}$ will be improved and we will establish various upper bounds on the solutions. We start by recalling the following Poincaré inequality:

$$
\lambda_{0}\|\mathbf{A}\|_{\mathbf{H}^{1}}^{2} \leq\|\nabla \times \mathbf{A}\|_{2}^{2}+\|\nabla \cdot \mathbf{A}\|_{2}^{2} \quad \forall \mathbf{A} \in \mathbf{H}^{1}(\Omega) \text { with }\left.\mathbf{n} \cdot \mathbf{A}\right|_{\partial \Omega}=0,
$$

where $\lambda_{0}$ is some positive constant.

The following lemma concerns the a priori $L^{2}$-bound of $\psi$ and $\mathbf{A}$.

Lemma 5.1. Assume the solution $u=(\psi, \mathbf{A})$ exists for all $t \geq 0$. Then there is a constant $C$ independent from $t, u$ and $\mathbf{H}$ such that for all $t \geq 0$

$$
\|\psi(t)\|_{2}^{2}+\|\mathbf{A}(t)\|_{2}^{2} \leq C\left[\mathrm{e}^{-\lambda_{0} t}\left(\left\|\psi_{0}\right\|_{2}^{2}+\left\|\mathbf{A}_{0}\right\|_{2}^{2}\right)+1+\int_{0}^{t} \mathrm{e}^{-\lambda_{0}(t-s)} \int_{\Omega}|\mathbf{H}(s, x)|^{2} \mathrm{~d} x \mathrm{~d} s\right],
$$

where $\lambda_{0}$ is given by (5.1).

Proof. The proof uses the following Green's identities:

$$
\int_{\Omega}(\nabla \cdot \mathbf{A}) \varphi \mathrm{d} x+\int_{\Omega} \mathbf{A} \cdot(\nabla \varphi) \mathrm{d} x=\int_{\partial \Omega}(\mathbf{n} \cdot \mathbf{A}) \varphi \mathrm{d} \sigma(x) \quad \forall \mathbf{A} \in \mathbf{H}(\operatorname{div} ; \Omega), \forall \varphi \in H^{1}(\Omega),
$$

where $\mathbf{H}(\operatorname{div} ; \Omega):=\left\{\mathbf{A} \in \mathbf{L}^{2}(\Omega): \nabla \cdot \mathbf{A} \in L^{2}(\Omega)\right\}$,

$$
\int_{\Omega}(\nabla \times \mathbf{A}) \cdot \mathbf{B} \mathrm{d} x-\int_{\Omega} \mathbf{A} \cdot(\nabla \times \mathbf{B}) \mathrm{d} x=\int_{\partial \Omega} \mathbf{B} \cdot(\mathbf{A} \times \mathbf{n}) \mathrm{d} \sigma(x)
$$

for all $\mathbf{A} \in \mathbf{H}(\operatorname{curl} ; \Omega):=\left\{\mathbf{A} \in \mathbf{L}^{2}(\Omega): \nabla \times \mathbf{A} \in \mathbf{L}^{2}(\Omega)\right\}$ and $\mathbf{B} \in \mathbf{H}^{1}(\Omega)$.

Multiplying equation (1.6) by $\psi^{*}$, integrating over $\Omega$ and taking the real part, we have

$$
\frac{\eta}{2} \frac{\mathrm{d}\|\psi\|_{2}^{2}}{\mathrm{~d} t}=-\frac{1}{\kappa^{2}} \int_{\partial \Omega} \gamma|\psi|^{2} \mathrm{~d} \sigma(x)-\left\|\left(\frac{i}{\kappa} \nabla+\mathbf{A}\right) \psi\right\|_{2}^{2}+\|\psi\|_{2}^{2}-\|\psi\|_{4}^{4}
$$

On the other hand taking the inner product of (1.7) with A yields

$$
\frac{1}{2} \frac{\mathrm{d}\|\mathbf{A}\|_{2}^{2}}{\mathrm{~d} t}=-\|\nabla \times \mathbf{A}\|_{2}^{2}-\|\nabla \cdot \mathbf{A}\|_{2}^{2}+\int_{\Omega} \mathbf{A} \cdot \mathbf{J}_{s} \mathrm{~d} x+\int_{\Omega} \mathbf{H} \cdot(\nabla \times \mathbf{A}) \mathrm{d} x
$$

The last two terms on the right hand side of (5.6) can be estimated as follows. Let $\varepsilon>0$, since $\|\psi(t)\|_{\infty} \leq 1$ for all $t \geq 0$ we replace $\mathbf{J}_{s}$ in (1.3) and use Young's inequality to obtain

$$
\left|\int_{\Omega} \mathbf{A} \cdot \mathbf{J}_{s} \mathrm{~d} x\right| \leq \frac{\varepsilon}{2}\|\mathbf{A}\|_{2}^{2}+\frac{1}{2 \varepsilon}\left\|\left(\frac{i}{\kappa} \nabla+\mathbf{A}\right) \psi\right\|_{2}^{2}
$$


and likewise

$$
\left|\int_{\Omega} \mathbf{H} \cdot(\nabla \times \mathbf{A}) \mathrm{d} x\right| \leq \frac{\varepsilon}{2}\|\nabla \times \mathbf{A}\|_{2}^{2}+\frac{1}{2 \varepsilon}\|\mathbf{H}\|_{2}^{2}
$$

Multiplying (5.6) by $\varepsilon$, adding to (5.5) and using Poincaré inequality (5.1) yield

$$
\begin{aligned}
& \frac{1}{2} \frac{\mathrm{d}}{\mathrm{d} t}\left(\eta\|\psi\|_{2}^{2}+\varepsilon\|\mathbf{A}\|_{2}^{2}\right) \\
& \quad \leq-\frac{1}{2}\left\|\left(\frac{i}{\kappa} \nabla+\mathbf{A}\right) \psi\right\|_{2}^{2}+\varepsilon\left(\varepsilon-\lambda_{0}\right)\|\mathbf{A}\|_{\mathbf{H}^{1}}^{2}+\|\psi\|_{2}^{2}+\frac{1}{2}\|\mathbf{H}\|_{2}^{2} .
\end{aligned}
$$

Thus

$$
\frac{1}{2} \frac{\mathrm{d}}{\mathrm{d} t}\left(\eta\|\psi\|_{2}^{2}+\varepsilon\|\mathbf{A}\|_{2}^{2}\right) \leq \varepsilon\left(\varepsilon-\lambda_{0}\right)\|\mathbf{A}\|_{\mathbf{H}^{1}}^{2}+|\Omega|+\frac{1}{2}\|\mathbf{H}\|_{2}^{2} .
$$

Now set $\chi(t)=\eta\|\psi(t)\|_{2}^{2}+\varepsilon\|\mathbf{A}(t)\|_{\mathbf{H}^{1}}^{2}$, we compute

$$
\frac{\mathrm{d} \chi}{\mathrm{d} t}(t)+\lambda_{0} \chi(t) \leq \varepsilon\left(2 \varepsilon-\lambda_{0}\right)\|\mathbf{A}(t)\|_{\mathbf{H}^{1}}^{2}+|\Omega|\left(2+\lambda_{0} \eta\right)+\|\mathbf{H}(t)\|_{2}^{2} .
$$

Therefore, after multiplying by $\mathrm{e}^{\lambda_{0} t}$ and integrating over $[0, T]$, we derive

$$
\chi(t) \leq \mathrm{e}^{-\lambda_{0} t} \chi(0)+\frac{|\Omega|\left(2+\lambda_{0} \eta\right)}{\lambda_{0}}+\int_{0}^{t} \mathrm{e}^{\lambda_{0}(s-t)} \|\left.\mathbf{H}(s)\right|_{2} ^{2} \mathrm{~d} s,
$$

where $\varepsilon$ is to be taken such that $0<\varepsilon<\lambda_{0} / 2$, which then implies (5.2) and completes the proof.

Remark that if we assume

$$
\sup _{t \geq 0} \int_{0}^{t} \int_{\Omega} \mathrm{e}^{-\lambda_{0}(t-s)}|\mathbf{H}(s, x)|^{2} \mathrm{~d} s \mathrm{~d} x<\infty,
$$

then the solutions are bounded in the sense of $L^{2}$-norm.

The following lemma establishes an estimate on the gradient of the vector potential $\mathbf{A}$.

Lemma 5.2. Assume $\psi$ and $\mathbf{A}$ exist for all $t \geq 0$. Then there is a constant $C$ independent from $t, \psi, \mathbf{A}$ and $\mathbf{H}$ such that for all $t \geq 0$

$$
\|\mathbf{A}(t)\|_{\mathbf{H}^{1}}^{2} \leq C\left[\mathrm{e}^{-\varepsilon_{0} t}\left\|\mathbf{A}_{0}\right\|_{\mathbf{H}^{1}}^{2}+1+\sup _{t \geq 0}\|\mathbf{H}(t)\|_{2}^{2}+\int_{0}^{t} \mathrm{e}^{-\varepsilon_{0}(t-s)}\left\|\frac{\partial \mathbf{H}}{\partial t}(s)\right\|_{2}^{2} \mathrm{~d} s\right],
$$

for some $\varepsilon_{0}>0$ small enough.

The proof uses the following Gronwall's type inequality.

Claim 5.3. Let $f$ be an absolutely continuous function on $[0, T]$, not necessary positive, such that

$$
f^{\prime}(t) \leq g(t) f(t)+h(t) \quad \text { a.e } t \in[0, T]
$$


where $g$ and $h$ are integrable functions on $[0, T]$. Then for all $\tau \in[0, T]$

$$
f(t) \leq \mathrm{e}^{\left(\int_{\tau}^{t} g(s) \mathrm{d} s\right)}\left[f(\tau)+\int_{\tau}^{t} h(s)\left(\mathrm{e}^{-\int_{\tau}^{s} g(r) \mathrm{d} r}\right) \mathrm{d} s\right] \quad \forall t \in[\tau, T] .
$$

Proof. Set $k(t)=f(t) \mathrm{e}^{-\int_{\tau}^{t} g(s) \mathrm{d} s}$. We have

$$
\begin{aligned}
k^{\prime}(t) & =\mathrm{e}^{-\int_{\tau}^{t} g(s) \mathrm{d} s}\left(f^{\prime}(t)-g(t) f(t)\right) \\
& \leq \mathrm{e}^{-\int_{\tau}^{t} g(s) \mathrm{d} s} h(t) .
\end{aligned}
$$

Therefore, integrating over $[\tau, t]$, we obtain for all $t \in[\tau, T]$

$$
f(t) \mathrm{e}^{-\int_{\tau}^{t} g(s) \mathrm{d} s}-f(\tau) \leq \int_{\tau}^{t} h(s) \mathrm{e}^{-\int_{\tau}^{s} g(r) \mathrm{d} r} \mathrm{~d} s,
$$

for all $\tau \in[0, T]$, which implies (5.15).

Proof of Lemma 5.2. Taking the inner product of (1.7) with $\partial \mathbf{A} / \partial t$ and using the identities (5.3)-(5.4) and the boundary conditions (1.8), we get

$$
\begin{aligned}
\frac{1}{2} \frac{\mathrm{d}}{\mathrm{d} t}\left(\|\nabla \times \mathbf{A}\|_{2}^{2}+\|\nabla \cdot \mathbf{A}\|_{2}^{2}\right)= & \int_{\Omega}\left(\frac{\partial \mathbf{A}}{\partial t}\right) \cdot(\nabla \times \mathbf{H}) \mathrm{d} x-\left\|\frac{\partial \mathbf{A}}{\partial t}\right\|_{2}^{2}+\int_{\Omega} \mathbf{J}_{s} \cdot\left(\frac{\partial \mathbf{A}}{\partial t}\right) \mathrm{d} x \\
= & \frac{\mathrm{d}}{\mathrm{d} t}\left(\int_{\Omega} \mathbf{H} \cdot(\nabla \times \mathbf{A}) \mathrm{d} x\right)-\int_{\Omega}\left(\frac{\partial \mathbf{H}}{\partial t}\right) \cdot(\nabla \times \mathbf{A}) \mathrm{d} x \\
& -\left\|\frac{\partial \mathbf{A}}{\partial t}\right\|_{2}^{2}+\int_{\Omega} \mathbf{J}_{s} \cdot\left(\frac{\partial \mathbf{A}}{\partial t}\right) \mathrm{d} x .
\end{aligned}
$$

Let $\varepsilon>0$ small enough. Using Young's inequality, it follows

$$
\begin{gathered}
\left|\int_{\Omega}\left(\frac{\partial \mathbf{H}}{\partial t}\right) \cdot(\nabla \times \mathbf{A}) \mathrm{d} x\right| \leq \frac{\varepsilon}{2}\|\nabla \times \mathbf{A}\|_{2}^{2}+\frac{1}{2 \varepsilon}\left\|\frac{\partial \mathbf{H}}{\partial t}\right\|_{2}^{2}, \\
\left|\int_{\Omega} \mathbf{J}_{s} \cdot\left(\frac{\partial \mathbf{A}}{\partial t}\right) \mathrm{d} x\right| \leq \frac{1}{2}\left\|\frac{\partial \mathbf{A}}{\partial t}\right\|_{2}^{2}+\frac{1}{2}\left\|\left(\frac{i}{\kappa} \nabla+\mathbf{A}\right) \psi\right\|_{2}^{2}
\end{gathered}
$$

Hence

$$
\begin{aligned}
\frac{1}{2} \frac{\mathrm{d}}{\mathrm{d} t}( & \left.\|\nabla \times \mathbf{A}\|_{2}^{2}+\|\nabla \cdot \mathbf{A}\|_{2}^{2}-2 \int_{\Omega} \mathbf{H} \cdot(\nabla \times \mathbf{A}) \mathrm{d} x\right) \\
\leq & \frac{1}{2}\left\|\left(\frac{i}{\kappa} \nabla+\mathbf{A}\right) \psi\right\|_{2}^{2}+\frac{\varepsilon}{2}\|\nabla \times \mathbf{A}\|_{2}^{2}+\frac{1}{2 \varepsilon}\left\|\frac{\partial \mathbf{H}}{\partial t}\right\|_{2}^{2}
\end{aligned}
$$

Multiplying (5.20) by $\varepsilon>0$ and adding to estimate (5.9) yield

$$
\begin{gathered}
\frac{1}{2} \frac{\mathrm{d}}{\mathrm{d} t}\left[\eta\|\psi\|_{2}^{2}+\varepsilon\left(\|\mathbf{A}\|_{2}^{2}+\|\nabla \times \mathbf{A}\|_{2}^{2}+\|\nabla \cdot \mathbf{A}\|_{2}^{2}\right)-2 \varepsilon \int_{\Omega} \mathbf{H} \cdot(\nabla \times \mathbf{A}) \mathrm{d} x\right] \\
\leq\left(\frac{3}{2} \varepsilon^{2}-\varepsilon \lambda_{0}\right)\|\mathbf{A}\|_{\mathbf{H}^{1}}^{2}+|\Omega|+\frac{1}{2}\left(\|\mathbf{H}\|_{2}^{2}+\left\|\frac{\partial \mathbf{H}}{\partial t}\right\|_{2}^{2}\right) .
\end{gathered}
$$


Put

$$
\theta(t)=\eta\|\psi\|_{2}^{2}+\varepsilon\left(\|\mathbf{A}\|_{2}^{2}+\|\nabla \times \mathbf{A}\|_{2}^{2}+\|\nabla \cdot \mathbf{A}\|_{2}^{2}\right)-2 \varepsilon \int_{\Omega} \mathbf{H} \cdot(\nabla \times \mathbf{A}) \mathrm{d} x .
$$

Under these circumstances, we have

$$
\theta^{\prime}(t)+\varepsilon \theta(t) \leq\left(5 \varepsilon^{2}-2 \varepsilon \lambda_{0}\right)\|\mathbf{A}\|_{\mathbf{H}^{1}}^{2}+(2+\varepsilon \eta)|\Omega|+2\|\mathbf{H}\|_{2}^{2}+\left\|\frac{\partial \mathbf{H}}{\partial t}\right\|_{2}^{2}
$$

Therefore by choosing $0<\varepsilon=\varepsilon_{0}<2 \lambda_{0} / 5$ and using Claim 5.3, we infer

$$
\begin{aligned}
\theta(t) & \leq \mathrm{e}^{-\varepsilon_{0} t}\left[\theta(0)+\int_{0}^{t} \mathrm{e}^{\varepsilon_{0} s}\left(\left(2+\varepsilon_{0} \eta\right)|\Omega|+2\|\mathbf{H}(s)\|_{2}^{2}+\left\|\frac{\partial \mathbf{H}}{\partial t}(s)\right\|_{2}^{2}\right) \mathrm{d} s\right] \\
& \leq \mathrm{e}^{-\varepsilon_{0} t} \theta(0)+\frac{2+\varepsilon_{0} \eta}{\varepsilon_{0}}|\Omega|+\frac{2}{\varepsilon_{0}} \sup _{t \geq 0}\|\mathbf{H}(t)\|_{2}^{2}+\int_{0}^{t} \mathrm{e}^{-\varepsilon_{0}(t-s)}\left\|\frac{\partial \mathbf{H}}{\partial t}(s)\right\|_{2}^{2} \mathrm{~d} s .
\end{aligned}
$$

Consequently (5.13) follows by substituting in $\theta(t)$ and using Hölder inequality, which completes the proof.

We continue with the estimate on the gradient of $\psi$.

Theorem 5.4. Provided $u$ exists for all $t \geq 0$, there is a constant $C$ independent from $t, u_{0}$, $u$ and $\mathbf{H}$ such that for all $t \geq 0$

$$
\|u(t)\|_{\mathbb{Q}^{1}}^{2} \leq C\left[\mathrm{e}^{-\varepsilon_{0} t}\left\|u_{0}\right\|_{\mathbb{Q}^{1}}^{2}+1+\sup _{t \geq 0}\|\mathbf{H}(t)\|_{\mathbf{H}^{1}}^{2}+\int_{0}^{t} \mathrm{e}^{-\varepsilon_{0}(t-s)}\left\|\frac{\partial \mathbf{H}}{\partial t}(s)\right\|_{2}^{2} \mathrm{~d} s\right]
$$

for some $\varepsilon_{0}>0$ small enough.

Proof. The proof of Theorem 5.4 uses the energy functional $E_{\omega}$ given by (3.29). Since the pair $(\psi, \mathbf{A})$ are weak solutions of the gauged TDGL equations (1.6)-(1.9) with $\omega=1$, the time derivative of $E_{1}(t)=E_{1}(t, \psi(t), \mathbf{A}(t))$ is

$$
\begin{aligned}
\frac{\mathrm{d} E_{1}}{\mathrm{~d} t}(t)= & -2 \int_{\Omega}\left[\eta\left|\frac{\partial \psi}{\partial t}-i \kappa \psi(\nabla \cdot \mathbf{A})\right|^{2}+\left|\frac{\partial \mathbf{A}}{\partial t}\right|^{2}+|\nabla(\nabla \cdot \mathbf{A})|^{2}\right] \mathrm{d} x \\
& -2 \int_{\Omega} \frac{\partial \mathbf{H}}{\partial t} \cdot(\nabla \times \mathbf{A}-\mathbf{H}) \mathrm{d} x,
\end{aligned}
$$

which implies

$$
\frac{\mathrm{d} E_{1}}{\mathrm{~d} t}(t) \leq-2 \int_{\Omega} \frac{\partial \mathbf{H}}{\partial t} \cdot(\nabla \times \mathbf{A}-\mathbf{H}) \mathrm{d} x
$$

Adding $E_{1}(t)$ to equation (5.27), we obtain

$$
\frac{\mathrm{d} E_{1}}{\mathrm{~d} t}(t)+E_{1}(t) \leq\left\|\left(\frac{i}{\kappa} \nabla+\mathbf{A}\right) \psi\right\|_{2}^{2}+C_{1}\left(\|\mathbf{A}\|_{\mathbf{H}^{1}}^{2}+\|\mathbf{H}\|_{2}^{2}+\left\|\frac{\partial \mathbf{H}}{\partial t}\right\|_{2}^{2}+1\right),
$$

with $C_{1}$ being a positive constant independent from $t, u$ and $\mathbf{H}$. 
Set $\xi(t)=\eta\|\psi(t)\|_{2}^{2}+\varepsilon\|\mathbf{A}(t)\|_{2}^{2}+\varepsilon^{2} E_{1}(t)$ with $\varepsilon>0$ small enough. Then by (5.9) and (5.29), we may write for $\varepsilon$ small enough

$$
\frac{\mathrm{d} \xi}{\mathrm{d} t}(t)+\varepsilon \xi(t) \leq C_{2}\left(1+\|\mathbf{H}\|_{2}^{2}+\left\|\frac{\partial \mathbf{H}}{\partial t}\right\|_{2}^{2}\right)
$$

with $C_{2}$ is independent from $t, u$ and $\mathbf{H}$.

Applying Claim 5.3, we infer

$$
\xi(t) \leq C_{3}\left(\mathrm{e}^{-\varepsilon t} \xi(0)+1+\sup _{t \geq 0}\|\mathbf{H}(t)\|_{2}^{2}+\int_{0}^{t} \mathrm{e}^{-\varepsilon(t-s)}\left\|\frac{\partial \mathbf{H}}{\partial t}(s)\right\|_{2}^{2} \mathrm{~d} s\right) .
$$

Consequently, we derive (5.26) by substituting in $E_{1}(t)$ and using (5.13). This concludes the proof of Theorem 5.4.

Remark 5.5. Let $\tau \geq 0$. According to Claim 5.3, a simple modification in the proofs of Lemma 5.1, Lemma 5.2, and Theorem 5.4 yields

$$
\|u(t)\|_{\mathbb{R}^{1}}^{2} \leq C\left[\mathrm{e}^{-\varepsilon_{0}(t-\tau)}\|u(\tau)\|_{\mathbb{M}^{1}}^{2}+1+\sup _{\tau \leq s \leq t}\|\mathbf{H}(s)\|_{\mathbf{H}^{1}}^{2}+\int_{\tau}^{t} \mathrm{e}^{-\varepsilon_{0}(t-s)}\left\|\frac{\partial \mathbf{H}}{\partial t}(s)\right\|_{2}^{2} \mathrm{~d} s\right],
$$

for all $t \geq \tau$, where $C$ is independent from $t, \tau, u$ and $\mathbf{H}$.

As a consequence of Remark 5.5, we derive global existence and uniform boundedness in time.

Corollary 5.6. For some $T>0$, we assume

$$
\begin{gathered}
\mathbf{H} \in L^{\infty}\left(0, \infty ; \mathbf{L}^{2}(\Omega)\right), \quad \frac{\partial \mathbf{H}}{\partial t} \in L^{2}\left(\tau, T ; \mathbf{L}^{2}(\Omega)\right) \quad \forall \tau>0, \\
\lim _{t \rightarrow 0^{+}}\left(t^{\mu}\left\|\frac{\partial \mathbf{H}}{\partial t}(t)\right\|_{2}\right)=0 \quad \text { for some } \mu<1 .
\end{gathered}
$$

Assume, moreover, that for some $0 \leq \tau_{0} \leq T$

$$
\limsup _{t \rightarrow+\infty} \int_{\tau_{0}}^{t} \int_{\Omega} \mathrm{e}^{-\varepsilon_{0}(t-s)}\left|\frac{\partial \mathbf{H}}{\partial t}(s, x)\right|^{2} \mathrm{~d} x \mathrm{~d} s<\infty,
$$

where $\varepsilon_{0}>0$ is given by Lemma 5.2 and Theorem 5.4. Then the solution $u=(\psi, \mathbf{A})$ is defined for all $t \geq 0$ and

$$
\|u(t)\|_{\mathbb{Q}^{1}} \leq C\left(\mathrm{e}^{-\varepsilon_{0}\left(t-\tau_{0}\right)}\left\|u\left(\tau_{0}\right)\right\|_{\mathbb{R}^{1}}+1\right) \quad \forall t \geq \tau_{0}
$$

where $C$ is a positive constant independent from $t$ and $u$. 
Remark 5.7. Since $u$ is continuous on $[0, T]$, (5.36) then implies

$$
\sup _{t \geq 0}\|u(t)\|_{\mathbb{R}^{1}}<\infty .
$$

In particular, if $\mathbf{H} \in L^{\infty}\left(0, \infty ; \mathbf{L}^{2}(\Omega)\right)$ and $\partial \mathbf{H} / \partial t \in L^{2}\left(0, \infty ; \mathbf{L}^{2}(\Omega)\right) \cup L^{\infty}\left(0, \infty ; \mathbf{L}^{2}(\Omega)\right)$, inequality (5.26) then becomes

$$
\|u(t)\|_{\mathbb{R}^{1}} \leq C\left(\mathrm{e}^{-\varepsilon_{0} t}\left\|u_{0}\right\|_{\mathbb{Q}^{1}}+1\right) \quad \forall t \geq 0 .
$$

The constant $C$ depends only on $\mathbf{H}$. More precisely,

$$
\limsup _{t \rightarrow+\infty}\|u(t)\|_{\mathbb{Q} \mathbb{1}^{1}} \leq C \quad \text { uniformly for }\left\|u_{0}\right\|_{\mathbb{Q} \mathbb{1}^{1}} \leq R,
$$

with $R>0$ arbitrary.

Remark 5.8. Clearly, Theorem 5.4 and Corollary 5.6 improve the result established in [22] (cf. Theorem 4.1). Furthermore, if we compare Corollary 5.6 with the corresponding results on global existence in [6] and [17], we see that our case does not assume integrability of $\partial \mathbf{H} / \partial t$ in a neighborhood of $t=0$.

TheOREM 5.9. Under the assumption

$$
\mathbf{H} \in L^{\infty}\left(0, \infty ; \mathbf{L}^{2}(\Omega)\right), \quad \frac{\partial \mathbf{H}}{\partial t} \in L^{2}\left(0, \infty ; \mathbf{L}^{2}(\Omega)\right) \cup L^{\infty}\left(0, \infty ; \mathbf{L}^{2}(\Omega)\right),
$$

we have for any pair of solutions $u_{1}=\left(\psi_{1}, \mathbf{A}_{1}\right)$ and $u_{2}=\left(\psi_{2}, \mathbf{A}_{2}\right)$ of equations (1.6)-(1.9) having as initial data $u_{1,0}$ and $u_{2,0}$

$$
\left\|u_{1}(t)-u_{2}(t)\right\|_{2} \leq \mathrm{e}^{C t}\left\|u_{1,0}-u_{2,0}\right\|_{2} \quad \forall t \geq 0
$$

where $C$ is a positive constant depending on $\mathbf{H},\left\|u_{1,0}\right\|_{\mathbb{a}^{1}}$ and $\left\|u_{2,0}\right\|_{\mathbb{\sharp}^{1}}$.

Proof. We have

$$
\begin{aligned}
\frac{\partial}{\partial t}\left(\psi_{1}-\psi_{2}\right)= & \frac{1}{\kappa^{2}} \Delta\left(\psi_{1}-\psi_{2}\right)+\left(\Gamma\left(t, \psi_{1}, \mathbf{A}_{1}^{\prime}\right)-\Gamma\left(t, \psi_{2}, \mathbf{A}_{2}^{\prime}\right)\right), \\
\frac{\partial}{\partial t}\left(\mathbf{A}_{1}^{\prime}-\mathbf{A}_{2}^{\prime}\right)= & -\nabla \times \nabla \times\left(\mathbf{A}_{1}^{\prime}-\mathbf{A}_{2}^{\prime}\right)+\nabla\left(\nabla \cdot\left(\mathbf{A}_{1}^{\prime}-\mathbf{A}_{2}^{\prime}\right)\right)+\mathbf{J}_{s}\left(\psi_{1}, \mathbf{A}_{1}^{\prime}\right) \\
& -\mathbf{J}_{s}\left(\psi_{2}, \mathbf{A}_{2}^{\prime}\right)-\mathbf{A}_{\mathbf{H}}\left(\left|\psi_{1}\right|^{2}-\left|\psi_{2}\right|^{2}\right),
\end{aligned}
$$

where $\Gamma$ is given by the expression

$\Gamma(t, \varphi, \mathbf{B})=\frac{1}{\eta}\left[-\frac{2 i}{\kappa}(\nabla \varphi) \cdot\left(\mathbf{B}+\mathbf{A}_{\mathbf{H}}\right)-\frac{i}{\kappa}\left(1-\eta \kappa^{2} \omega\right) \varphi(\nabla \cdot \mathbf{B})-\varphi\left|\mathbf{B}+\mathbf{A}_{\mathbf{H}}\right|^{2}+\left(1-|\varphi|^{2}\right) \varphi\right]$,

for all $(\varphi, \mathbf{B}) \in \mathscr{H}^{1}(\Omega) \times \mathbf{H}^{1}(\Omega)$. $\mathbf{J}_{s}$ being given by $(1.3)$. 
On one hand, multiplying (5.42) by $\left(\psi_{1}-\psi_{2}\right)^{*}$, integrating over $\Omega$ and taking the real part yields

$$
\begin{aligned}
\frac{\eta}{2} \frac{\mathrm{d}}{\mathrm{d} t}\left\|\psi_{1}-\psi_{2}\right\|_{2}^{2}= & -\frac{1}{\kappa^{2}}\left\|\nabla\left(\psi_{1}-\psi_{2}\right)\right\|_{2}^{2}+\frac{1}{\kappa^{2}} \int_{\partial \Omega} \gamma(x)\left|\psi_{1}-\psi_{2}\right|^{2} \mathrm{~d} \sigma(x) \\
& +\int_{\Omega}\left[\Gamma\left(t, \psi_{1}, \mathbf{A}_{1}^{\prime}\right)-\Gamma\left(t, \psi_{2}, \mathbf{A}_{2}^{\prime}\right)\right]\left(\psi_{1}-\psi_{2}\right)^{*} \mathrm{~d} x .
\end{aligned}
$$

On the other hand, multiplying (5.43) by $\mathbf{A}_{1}^{\prime}-\mathbf{A}_{2}^{\prime}$, integrating over $\Omega$ and using (5.1), we get

$$
\begin{aligned}
& \frac{1}{2} \frac{\mathrm{d}}{\mathrm{d} t}\left(\left\|\mathbf{A}_{1}^{\prime}-\mathbf{A}_{2}^{\prime}\right\|_{2}^{2}\right)+\lambda_{0}\left\|\mathbf{A}_{1}^{\prime}-\mathbf{A}_{2}^{\prime}\right\|_{\mathbf{H}^{1}}^{2} \\
& \quad \leq \int_{\Omega}\left(\mathbf{A}_{1}^{\prime}-\mathbf{A}_{2}^{\prime}\right) \cdot\left[\mathbf{J}_{s}\left(\psi_{1}, \mathbf{A}_{1}^{\prime}\right)-\mathbf{J}_{s}\left(\psi_{2}, \mathbf{A}_{2}^{\prime}\right)-\mathbf{A}_{\mathbf{H}}\left(\left|\psi_{1}\right|^{2}-\left|\psi_{2}\right|^{2}\right)\right] \mathrm{d} x .
\end{aligned}
$$

$\left\|u_{1}(t)\right\|_{\mathbb{Q}_{1}}$ and $\left\|u_{2}(t)\right\|_{\mathbb{Q}_{1}^{1}}$ being bounded for all $t \geq 0$, we add (5.45) to (5.46) and use standard arguments, thus arriving at

$$
\frac{\mathrm{d}}{\mathrm{d} t}\left(\left\|u_{1}(t)-u_{2}(t)\right\|_{2}^{2}\right) \leq C\left\|u_{1}(t)-u_{2}(t)\right\|_{2}^{2} \quad \forall t \geq 0,
$$

where $C$ is a positive constant independent from $t$. It depends on $\mathbf{H},\left\|u_{1,0}\right\|_{\mathbb{A}^{1}}$ and $\left\|u_{2,0}\right\|_{\mathbb{Q} 1}$. Therefore we may apply Gronwall's inequality to conclude

$$
\left\|u_{1}(t)-u_{2}(t)\right\|_{2}^{2} \leq \mathrm{e}^{C t}\left\|u_{1,0}-u_{2,0}\right\|_{2}^{2} \quad \forall t \geq 0
$$

Remark 5.10. Inequality (5.41) confirms the uniqueness of the solutions with respect to initial data. Furthermore, Theorem 5.9 extends the result in [15] (cf. Theorem 3.1) to the nonstationary case.

\section{References}

[1] A. Abrikosov, Fundamentals of the Theory of Metals, North-Holland, Amsterdam, 1988.

[2] J. Bardeen, L. N. Cooper, and J. R. Schrieffer, Theory of superconductivity, Phys. Rev. (2) 108 (1957), 1175-1204.

[3] R. Dautray and J.-L. Lions, Mathematical Analysis and Numerical Methods for Science and Technology. Vol. 5. Evolution Problems. I, Springer-Verlag, Berlin, 1992.

[4] Q. Du, Global existence and uniqueness of solutions of the time-dependent Ginzburg-Landau model for superconductivity, Appl. Anal. 53 (1994), no. 1-2, 1-17.

[5] J. Fleckinger-Pellé and H. G. Kaper, Gauges for the Ginzburg-Landau equations of superconductivity, ZAMM Z. Angew. Math. Mech. 76 (1996), no. 2, 345-348.

[6] J. Fleckinger-Pellé, H. G. Kaper, and P. Takáč, Dynamics of the Ginzburg-Landau equations of superconductivity, Nonlinear Anal. 32 (1998), no. 5, 647-665.

[7] V. L. Ginzburg and L. D. Landau, On the theory of superconductivity, Zh. Eksp. Teor. Fiz. (USSR) 20 (1950), 1064-1082 (Russian), English translation in D. Ter Haar and L. D. Landau, Men of Physics, Vol. I, Pergamon Press, Oxford, 1965, 138-167.

[8] L. P. Gor'kov and G. M. Eliashberg, Generalizations of the Ginzburg-Landau equations for nonstationary problems in the case of alloys with paramagnetic impurities, Zh. Eksp. Teor. Fiz. $\mathbf{5 4}$ (1968), 612-626 (Russian), Soviet Phys. JETP 27 (1968), 328-334. 
[9] J. K. Hale, Asymptotic Behavior of Dissipative Systems, Mathematical Surveys and Monographs, vol. 25, American Mathematical Society, Rhode Island, 1988.

[10] D. Henry, Geometric Theory of Semilinear Parabolic Equations, Lecture Notes in Mathematics, vol. 840, Springer-Verlag, Berlin, 1981.

[11] K.-H. Hoffmann and Q. Tang, Ginzburg-Landau Phase Transition Theory and Superconductivity, International Series of Numerical Mathematics, vol. 134, Birkhäuser Verlag, Basel, 2001.

[12] H. G. Kaper and P. Takáč, Ginzburg-Landau dynamics with a time-dependent magnetic field, Nonlinearity 11 (1998), no. 2, 291-305.

[13] J. Liang and Q. Tang, Asymptotic behaviour of the solutions of an evolutionary Ginzburg-Landau superconductivity model, J. Math. Anal. Appl. 195 (1995), no. 1, 92-107.

[14] A. Pazy, Semigroups of Linear Operators and Applications to Partial Differential Equations, Applied Mathematical Sciences, vol. 44, Springer-Verlag, New York, 1983.

[15] A. Rodriguez-Bernal, B. Wang, and R. Willie, Asymptotic behaviour of time-dependent Ginzburg-Landau equations of superconductivity, Math. Methods Appl. Sci. 22 (1999), no. $18,1647-1669$.

[16] G. R. Sell and Y. You, Dynamics of Evolutionary Equations, Applied Mathematical Sciences, vol. 143, Springer-Verlag, New York, 2002.

[17] P. Takáč, On the dynamical process generated by a superconductivity model, ZAMM Z. Angew. Math. Mech. 76 (1996), no. 2, 349-352.

[18] Q. Tang, On an evolutionary system of Ginzburg-Landau equations with fixed total magnetic flux, Comm. Partial Differential Equations 20 (1995), no. 1-2, 1-36.

[19] Q. Tang and S. Wang, Time dependent Ginzburg-Landau equations of superconductivity, Phys. D 88 (1995), no. 3-4, 139-166.

[20] R. Temam, Infinite-Dimensional Dynamical Systems in Mechanics and Physics, Applied Mathematical Sciences, vol. 68, Springer-Verlag, New York, 1988.

[21] M. Tinkham, Introduction to Superconductivity, McGraw-Hill, New York, 1975.

[22] F. Zaouch, Global existence and boundedness of solutions of the time-dependent Ginzburg-Landau equations with a time-dependent magnetic field, Rostock. Math. Kolloq. (2003), no. 57, 5370.

[23] Time-periodic solutions of the time-dependent Ginzburg-Landau equations of superconductivity, Z. Angew. Math. Phys. 54 (2003), no. 6, 905-918.

[24] E. Zeidler, Nonlinear Functional Analysis and Its Applications. I. Fixed-Point Theorems, SpringerVerlag, New York, 1986.

Fouzi Zaouch: Laboratoire de Mathématiques et Applications (L.M.A.), Pôle Sciences et Technologies, Université de La Rochelle, Avenue Michel Crépeau, 17042 La Rochelle Cedex, France

E-mail address: fouzi.zaouch@univ-lr.fr 


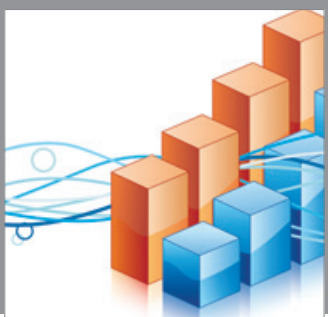

Advances in

Operations Research

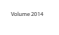

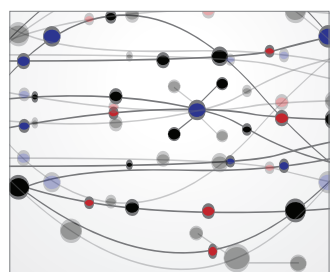

\section{The Scientific} World Journal
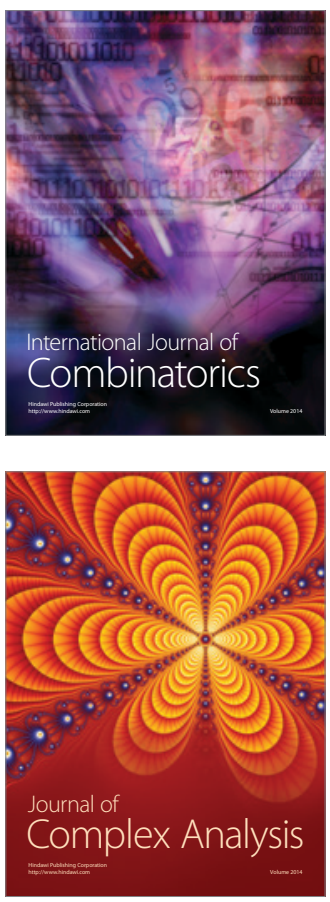

International Journal of

Mathematics and

Mathematical

Sciences
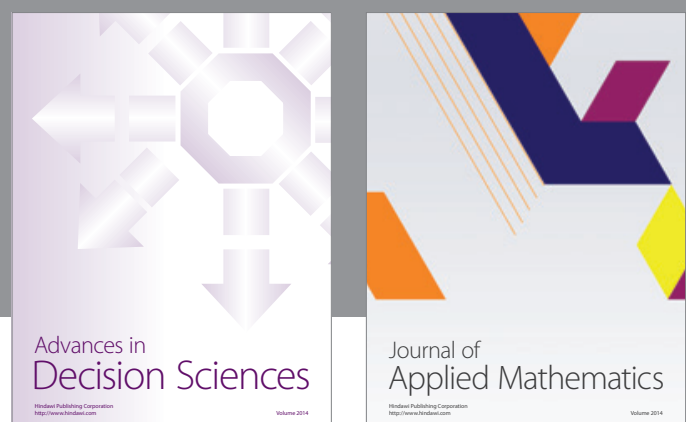

Journal of

Applied Mathematics
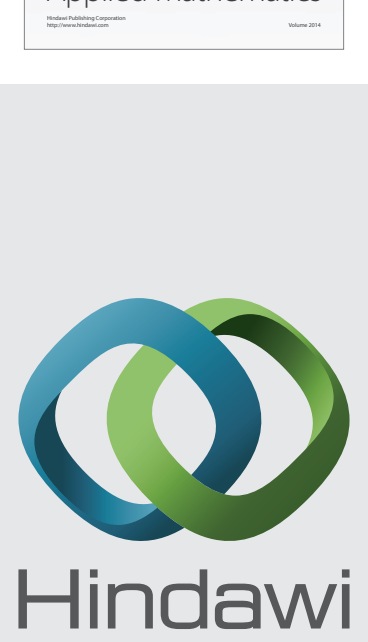

Submit your manuscripts at http://www.hindawi.com
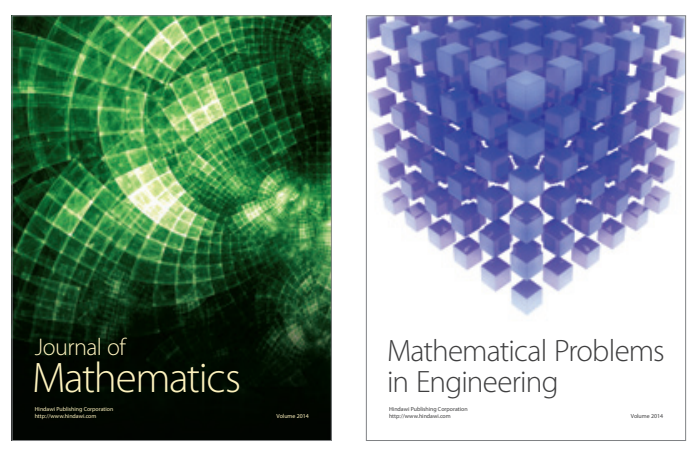

Mathematical Problems in Engineering
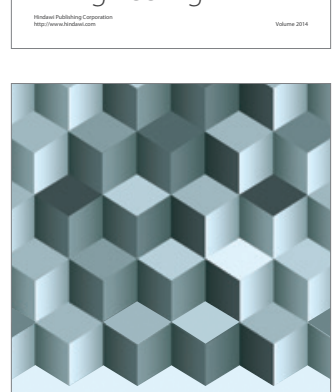

Journal of

Function Spaces
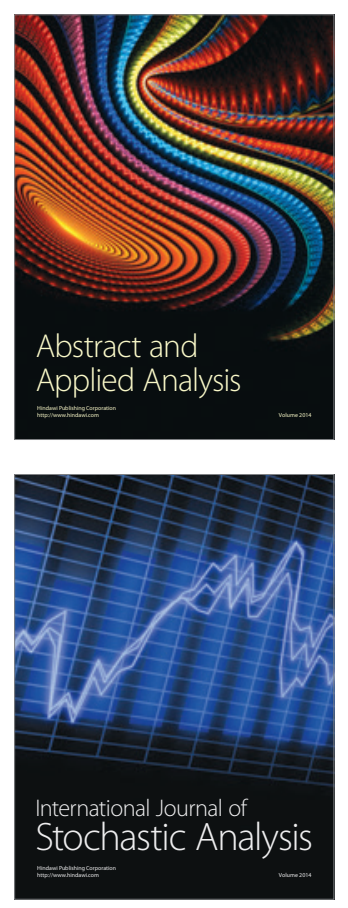

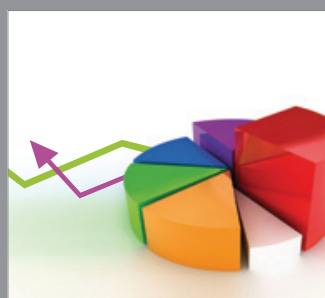

ournal of

Probability and Statistics

Promensencen
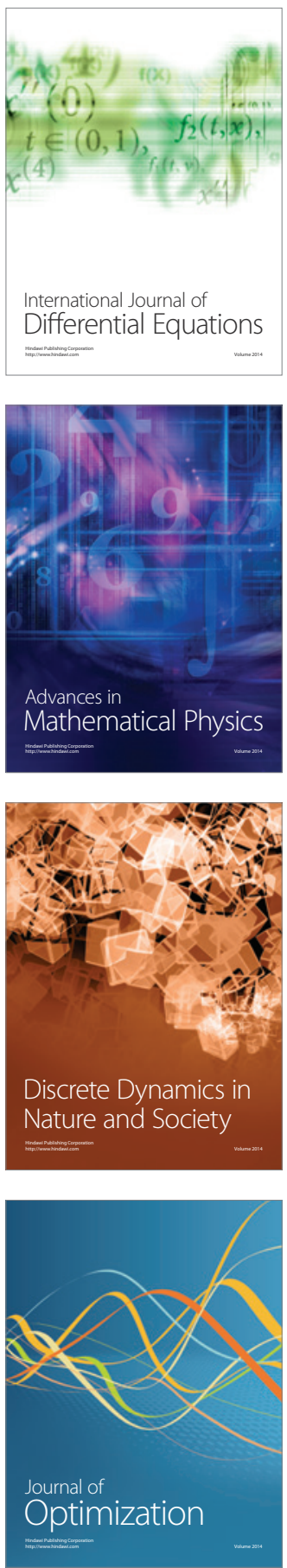